\title{
Supersymmetric Yang-Mills theory on conformal supergravity backgrounds in ten dimensions
}

\author{
Paul de Medeiros and José Figueroa-O'Farrill \\ Maxwell Institute and School of Mathematics, The University of Edinburgh, \\ James Clerk Maxwell Building, Peter Guthrie Tait Road, Edinburgh EH9 3FD, U.K. \\ E-mail: p.f.demedeiros@gmail.com, j.m.figueroa@ed.ac.uk
}

ABSTRACT: We consider bosonic supersymmetric backgrounds of ten-dimensional conformal supergravity. Up to local conformal isometry, we classify the maximally supersymmetric backgrounds, determine their conformal symmetry superalgebras and show how they arise as near-horizon geometries of certain half-BPS backgrounds or as a plane-wave limit thereof. We then show how to define Yang-Mills theory with rigid supersymmetry on any supersymmetric conformal supergravity background and, in particular, on the maximally supersymmetric backgrounds. We conclude by commenting on a striking resemblance between the supersymmetric backgrounds of ten-dimensional conformal supergravity and those of eleven-dimensional Poincaré supergravity.

KEYWords: Supergravity Models, Supersymmetric gauge theory, Differential and Algebraic Geometry

ARXiv EPRINT: 1512.08342 


\section{Contents}

1 Introduction 1

2 Conformal supergravity backgrounds $\quad 4$

2.1 Conformal gravity supermultiplet 4

2.2 Supersymmetric backgrounds 4

$\begin{array}{ll}2.3 \text { Conformal symmetry superalgebras } & 6\end{array}$

2.4 Maximally supersymmetric backgrounds $\quad 8$

$\begin{array}{lll}2.4 .1 & \text { Conformal symmetry superalgebras } & 10\end{array}$

$\begin{array}{lll}2.4 .2 & \mathfrak{s}\left(A d S_{3} \times S^{7}\right) \text { and } \mathfrak{s}\left(A d S_{7} \times S^{3}\right) & 11\end{array}$

$\begin{array}{lll}2.4 .3 & \mathfrak{s}\left(C W_{10}(A)\right) & 13\end{array}$

$\begin{array}{lll}2.4 .4 & \text { Contractions } & 14\end{array}$

$\begin{array}{ll}\text { 2.4.5 Maximal superalgebras } & 17\end{array}$

2.5 F1-string and NS5-brane, Weyl transformations and near-horizon limits 20

3 Yang-Mills supermultiplet $\quad 21$

4 Lifting to eleven dimensions $\quad \mathbf{2 3}$

4.1 Supersymmetric solutions in eleven dimensions 24

4.2 Kaluza-Klein embedding of supersymmetric type I backgrounds 24

4.3 Embedding of maximally supersymmetric Freund-Rubin backgrounds 25

$\begin{array}{ll}4.4 & \text { Branes, delocalisation and near-horizon limits } 26\end{array}$

$\begin{array}{ll}\text { A Clifford algebra and spinors in ten dimensions } & 28\end{array}$

\section{Introduction}

Supersymmetry multiplets in ten-dimensional spacetime not only underpin the five critical string theories (and their respective low-energy supergravity limits) but also encode the intricate structure of extended supersymmetry in many interesting quantum field theories in lower dimensions. For example, the Yang-Mills supermultiplet in ten dimensions elegantly captures the structure of extended supersymmetry and R-symmetry for gauge couplings in lower dimensions. Of course, in dimensions greater than four, even supersymmetric quantum field theories are not expected to be renormalisable without some kind of nonperturbative UV completion (indeed, this is precisely what string theory aims to provide). Without this completion, they should merely be regarded as low-energy effective field theories. 
In addition to the more familiar (gauged) type I, (Romans ${ }^{1}$ ) type IIA and type IIB Poincaré gravity supermultiplets [1-8] associated with critical string theory, there is also a conformal gravity supermultiplet in ten dimensions [9]. This conformal gravity supermultiplet can be gauged and the coupling described in [9] to a Yang-Mills supermultiplet in ten dimensions is reminiscent of the analogous Chapline-Manton [1] coupling for type I supergravity. Unlike the Poincaré supergravity theories in ten dimensions though, this conformal supergravity theory is manifestly off-shell and must be supplemented with some differential constraints in order to render it local. As a supergravity theory, it is therefore somewhat exotic but admits a consistent truncation to type I supergravity and reduces correctly to known extended conformal supergravity theories in both four and five dimensions. There is also a little conceptual deviation from the unextended conformal gravity supermultiplets in lower dimensions which result from gauging one of the conformal superalgebras on Nahm's list [10]. Of course, this is not surprising since there are no conformal superalgebras of the conventional type above dimension six. ${ }^{2}$ There do exist more general notions of a conformal superalgebra where the conformal algebra is contained in a less obvious manner. In particular, it was shown in [12] that the Lie superalgebra osp $(1 \mid 32)$ can be thought of as a conformal superalgebra for $\mathbb{R}^{9,1}$ with respect to a particular $\mathfrak{s o}(10,2)<\mathfrak{o s p}(1 \mid 32)$. Alas, it remains unclear though whether conformal supergravity in ten dimensions is somehow related to gauging this $\mathfrak{o s p}(1 \mid 32)$.

There is a vast literature on the classification of supersymmetric solutions of supergravity theories in diverse dimensions: that is to say, backgrounds which preserve some amount of rigid supersymmetry and solve the supergravity field equations. Indeed, at least for Poincaré supergravities, it is often the case that the preservation of a sufficient amount of rigid supersymmetry will guarantee that all of the supergravity field equations are satisfied. This typically comes from the so-called integrability conditions which result from iterating the 'Killing spinor' equations imposed by the preservation of supersymmetry.

In recent years, there has been mounting interest in the somewhat broader task of classifying supersymmetric backgrounds of conformal and Poincaré supergravity theories (which need not necessarily solve the field equations, only the integrability conditions). This is motivated primarily by a renewed curiosity in the general structure of quantum field theories with rigid supersymmetry in curved space [13-93], for which supersymmetric localisation has substantiated many important exact results and novel holographic applications $[18,20,28,31,34,47,52,53,55,57,67,78,85,87,88]$. The general strategy for obtaining non-trivial background geometries which support rigid supersymmetry builds on the pioneering work of Festuccia and Seiberg in four dimensions [15]. Given a rigid supermultiplet in flat space, it is often possible to promote it to a local supermultiplet in curved space via an appropriate supergravity coupling. For example, such a coupling

\footnotetext{
${ }^{1}$ This epithet is added when the zero-form RR flux in the type IIA gravity supermultiplet is non-zero.

${ }^{2}$ By this we mean that there exists no real Lie superalgebra obeying the axioms of [10] whose even part is of the form $\mathfrak{s o}(s+1, t+1) \oplus \mathfrak{R}$, for any real Lie algebra $\mathfrak{R}$, if $s+t>6$. Similarly, but with different hypotheses, for $n>6$, the maximal transitive prolongation of the $\mathbb{Z}$-graded complex Lie superalgebra $\mathfrak{h}=\mathfrak{h}_{-1} \oplus \mathfrak{h}_{-2}$, with $\mathfrak{h}_{-1}$ a (not necessary irreducible) spinor module of $\mathfrak{s o}_{n}(\mathbb{C})$ and $\mathfrak{h}-2=\mathbb{C}^{n}$, the vector representation, has no pieces in positive degree [11].
} 
can be induced holographically in a superconformal field theory in flat space that is dual to a string theory in an asymptotically anti-de Sitter background. A judicious choice of decoupling limit (in which the Planck mass becomes infinite) typically ensures that the dynamics of the gravity supermultiplet are effectively frozen out, leaving only the fixed bosonic supergravity fields as data encoding the geometry of the rigidly supersymmetric curved background.

The aim of this paper is to explore various aspects of bosonic supersymmetric backgrounds of conformal supergravity in ten dimensions and elucidate the structure of the rigid Yang-Mills supermultiplet on these backgrounds. In particular, we will classify the maximally supersymmetric conformal supergravity backgrounds, compute their associated conformal symmetry superalgebras and show how they are related to each other via certain algebraic limits. We will also show how to ascribe to any conformal supergravity background a conformal Killing superalgebra that is generated by its Killing spinors. Paying close attention to the non-trivial Weyl symmetry which acts within this class of conformal supergravity backgrounds, we will see how to recover the subclass of type I supergravity backgrounds and how certain Weyl-transformed versions of the half-BPS string and fivebrane backgrounds of type I supergravity recover, in the near-horizon limit, the maximally supersymmetric conformal supergravity backgrounds of Freund-Rubin type. We will then describe the rigid supersymmetry transformations and invariant lagrangian for the YangMills supermultiplet on any bosonic supersymmetric conformal supergravity background. This will be done both on-shell and in the partially off-shell formalism of [94-96]. We conclude with a curious observation that several highly supersymmetric conformal supergravity backgrounds in ten dimensions can be embedded in solutions of eleven-dimensional Poincaré supergravity which preserve twice as much supersymmetry.

This paper is organised as follows. In section 2 we discuss supersymmetric backgrounds of ten-dimensional conformal supergravity. In sections 2.1 and 2.2 we discuss the conformal gravity supermultiplet, the Killing spinor equation and its integrability condition. In section 2.3 we define the notion of a conformal symmetry superalgebra and show that every supersymmetric conformal supergravity background admits a conformal Killing superalgebra, which we define to be the ideal of a conformal symmetry superalgebra that is generated by the Killing spinors of the background. In section 2.4 we classify those conformal supergravity backgrounds preserving maximal supersymmetry. The results mimic those of eleven-dimensional supergravity: besides the (conformally) flat background, we have a pair of Freund-Rubin families and their plane-wave limit. In sections 2.4.1, 2.4.2 and 2.4.3 we work out the conformal symmetry superalgebras of these backgrounds and show in section 2.4.4 that the Killing superalgebra of the plane-wave limit arises as an Inönü-Wigner contraction of the Killing superalgebra of the Freund-Rubin backgrounds. In section 2.4.5 we comment on the maximal superalgebra of the maximally supersymmetric backgrounds, showing that it is isomorphic to osp $(1 \mid 16)$ for the Freund-Rubin backgrounds and non-existent for their plane-wave limit. In section 2.5 we discuss some of the halfBPS backgrounds of conformal supergravity and show how the Freund-Rubin maximally supersymmetric backgrounds arise as near-horizon geometries. In section 3 we introduce the on-shell Yang-Mills supermultiplet and write down a supersymmetric lagrangian on 
any supersymmetric conformal supergravity background. We then describe a partially offshell formulation of supersymmetric Yang-Mills theory on any such background. Finally, in section 4 we explore a possible relation between ten-dimensional conformal supergravity and eleven-dimensional Poincaré supergravity suggested by the resemblance between their maximally supersymmetric backgrounds and some of the half-BPS backgrounds of both theories. Appendix A contains our Clifford algebra conventions.

\section{Conformal supergravity backgrounds}

\subsection{Conformal gravity supermultiplet}

The off-shell conformal gravity supermultiplet in ten dimensions was constructed in [9]. The bosonic sector contains a metric $g_{\mu \nu}$, a six-form gauge potential $C_{\mu_{1} \ldots \mu_{6}}$ and an auxiliary scalar $\phi$. The fermionic sector contains a gravitino $\psi_{\mu}$ and an auxiliary spinor $\chi$. Both $\psi_{\mu}$ and $\chi$ are Majorana-Weyl spinor-valued, with opposite chiralities. ${ }^{3}$ The bosonic fields $g_{\mu \nu}$ and $C_{\mu_{1} \ldots \mu_{6}}$ contribute $44+84$ off-shell degrees of freedom, matching the $8 \times 16$ offshell degrees of freedom from the fermionic field $\psi_{\mu}$. The fields $\left(g_{\mu_{\nu}}, C_{\mu_{1} \ldots \mu_{6}}, \phi, \psi_{\mu}, \chi\right)$ are assigned Weyl weights $\left(2,0, w, \frac{1}{2},-\frac{1}{2}\right)$.

The supersymmetry variations for this theory can be found in equation (3.34) of [9] and must be supplemented with the constraint defined in their equation (3.35). Their ' $Q$ ' and ' $\mathrm{S}$ ' supersymmetry parameters are described by a pair of Majorana-Weyl spinors $\epsilon$ and $\eta$ with opposite chiralities: for definiteness, we shall take $\epsilon$ to have positive chirality, i.e., $\Gamma \epsilon=\epsilon$. A bosonic supersymmetric background of this theory follows by solving the equations obtained by setting to zero the combined ' $Q$ ' and ' $S$ ' supersymmetry variation of $\psi_{\mu}$ and $\chi$, evaluated at $\psi_{\mu}=0$ and $\chi=0$.

On a ten-dimensional lorentzian manifold $(M, g)$ equipped with Levi-Civita connection $\nabla$, the equations which follow from this procedure are

$$
\begin{aligned}
\nabla_{\mu} \epsilon+\frac{1}{4} \phi^{6 / w}\left(\Gamma_{\mu} K+2 K \Gamma_{\mu}\right) \epsilon & =\Gamma_{\mu} \eta \\
\frac{1}{2 w} \phi^{-1}(\not \varnothing \phi) \epsilon+\frac{1}{12} \phi^{6 / w} K \epsilon & =\eta
\end{aligned}
$$

where $K=\mathrm{d} C$. The constraint in equation (3.36) of [9] follows as an integrability condition from (2.1). Notice that the second equation in (2.1), derived from the supersymmetry variation of $\chi$, is simply a definition of $\eta$ in terms of the other background data. Substituting this definition into the first equation in (2.1) thus yields the defining condition for a bosonic supersymmetric background.

\subsection{Supersymmetric backgrounds}

Let us now define a more convenient set of background fields to work with:

$$
\Phi:=\frac{3}{w} \ln \phi \quad \text { and } \quad H:=4 \phi^{6 / w} \star K,
$$

\footnotetext{
${ }^{3}$ Our spinor conventions are contained in appendix A.
} 
and write $G=\mathrm{d} \Phi$. The three-form $H$ obeys d $\left(\mathrm{e}^{-2 \Phi} \star H\right)=0$ since $K$ is a closed seven-form. In terms of this data, the defining condition (2.1) for a bosonic supersymmetric background $(M, g, G, H)$ of conformal supergravity in ten dimensions becomes

$$
\nabla_{\mu} \epsilon=\frac{1}{6} \Gamma_{\mu} G \epsilon+\frac{1}{24} \Gamma_{\mu} H \epsilon+\frac{1}{8} H \Gamma_{\mu} \epsilon
$$

Under a Weyl transformation $g_{\mu \nu} \mapsto \Omega^{2} g_{\mu \nu}$, for some positive function $\Omega$, it follows that $\Gamma_{\mu} \mapsto \Omega \Gamma_{\mu}$ and $\epsilon \mapsto \sqrt{\Omega} \epsilon$. The condition (2.3) is therefore preserved under any such transformation provided $H \mapsto \Omega^{2} H$ and $\Phi \mapsto \Phi+3 \ln \Omega$. Consequently, performing this transformation with $\Omega=\mathrm{e}^{-\Phi / 3}$ allows one to fix $G=0$ in equation (2.3) with $H$ coclosed:

$$
\nabla_{\mu} \epsilon=\frac{1}{24} \Gamma_{\mu} H \epsilon+\frac{1}{8} H \Gamma_{\mu} \epsilon \quad \text { and } \quad \mathrm{d} \star H=0 .
$$

The condition (2.3) implies that the 'Dirac current' one-form $\xi_{\mu}=\bar{\epsilon} \Gamma_{\mu} \epsilon$ and the selfdual five-form $\zeta_{\mu \nu \rho \sigma \tau}=\bar{\epsilon} \Gamma_{\mu \nu \rho \sigma \tau} \epsilon$ obey

$$
\nabla_{\mu} \xi_{\nu}=\frac{1}{3} g_{\mu \nu} G_{\xi}+\frac{1}{3}\left(H_{\mu \nu \rho} \xi^{\rho}+2 G_{[\mu} \xi_{\nu]}+\frac{1}{12} \zeta_{\mu \nu \rho \sigma \tau} H^{\rho \sigma \tau}\right)
$$

and

$$
\nabla^{\tau} \zeta_{\mu \nu \rho \sigma \tau}=2 \zeta_{\mu \nu \rho \sigma \tau} G^{\tau}-4 H_{[\mu \nu \rho} \xi_{\sigma]}
$$

Taking the $(\mu \nu)$ symmetric part of $(2.5)$ implies $\mathscr{L}_{\xi} g=-2 \sigma_{\xi} g$ with

$$
\sigma_{\xi}=-\frac{1}{10} \nabla_{\mu} \xi^{\mu}=-\frac{1}{3} \mathscr{L}_{\xi} \Phi
$$

which shows that $\xi$ is a conformal Killing vector. Furthermore, acting with $\nabla^{\sigma}$ on (2.6) and using closure of $\mathrm{e}^{-2 \Phi} \star H$ and $G$ together with (2.5) and (2.6) on the right hand side implies

$$
\mathscr{L}_{\xi} H=-2 \sigma_{\xi} H
$$

If $H$ is closed then solutions of (2.3) with $G \epsilon=\frac{1}{2} H \epsilon$ describe bosonic supersymmetric backgrounds of type I supergravity in ten dimensions. In that case, (2.3) reduces to $\nabla_{\mu} \epsilon=$ $\frac{1}{8} H_{\mu \nu \rho} \Gamma^{\nu \rho} \epsilon, \xi$ is a Killing vector and $\iota_{\xi} H$ is closed. Clearly any such background is a special case of (2.3) and so one can always perform a Weyl transformation to obtain a solution of (2.4). However, if the original background had $G \neq 0$ then the new supersymmetric background of conformal supergravity solving (2.4) will no longer be a supersymmetric background of type I supergravity since the required Weyl transformation does not preserve the defining conditions $\mathrm{d} H=0$ and $G \epsilon=\frac{1}{2} H \epsilon$. 
Evaluating $\left[\nabla_{\mu}, \nabla_{\nu}\right] \epsilon$ implies the integrability condition

$$
\begin{aligned}
& \frac{1}{4}\left(R_{\mu \nu \rho \sigma}-\frac{2}{3} \nabla_{[\mu} H_{\nu] \rho \sigma}-\frac{1}{6} H_{\mu \rho \alpha} H_{\nu \sigma}{ }^{\alpha}-\frac{4}{9} H_{\mu \nu \rho} G_{\sigma}\right) \Gamma^{\rho \sigma} \epsilon+\frac{1}{18}\left(\frac{1}{24} H_{\alpha \beta \gamma} H^{\alpha \beta \gamma}-G_{\alpha} G^{\alpha}\right) \Gamma_{\mu \nu} \epsilon \\
& -\frac{1}{3}\left(\nabla_{[\mu} G^{\rho}+\frac{1}{3} G_{[\mu} G^{\rho}-\frac{1}{24} H_{\alpha \beta}^{\rho} H_{[\mu}^{\alpha \beta}-\frac{1}{3} G_{\alpha} H_{[\mu}{ }^{\rho \alpha}\right) \Gamma_{\nu] \rho} \epsilon+\frac{1}{36}\left(H^{\rho \sigma \alpha} G_{\alpha}\right) \Gamma_{\mu \nu \rho \sigma} \epsilon \\
& +\frac{1}{108}\left(H_{\mu \nu \alpha} H_{\beta \gamma \delta}+\frac{1}{16} H_{[\mu}^{\rho \sigma} \star H_{\nu] \rho \sigma \alpha \beta \gamma \delta}+\frac{1}{64} \varepsilon_{\mu \nu \alpha \beta \gamma \delta}^{\rho \sigma \theta \phi} H_{\rho \sigma \epsilon} H_{\theta \phi}{ }^{\epsilon}\right) \Gamma^{\alpha \beta \gamma \delta} \epsilon \\
& +\frac{1}{72}\left(\nabla_{\mu} H_{\alpha \beta \gamma}+H_{\mu \alpha \beta} G_{\gamma}+\frac{1}{3} H_{\alpha \beta \gamma} G_{\mu}-H_{\mu \alpha}{ }^{\rho} H_{\beta \gamma \rho}\right) \Gamma_{\nu}^{\alpha \beta \gamma} \epsilon \\
& -\frac{1}{72}\left(\nabla_{\nu} H_{\alpha \beta \gamma}+H_{\nu \alpha \beta} G_{\gamma}+\frac{1}{3} H_{\alpha \beta \gamma} G_{\nu}-H_{\nu \alpha}{ }^{\rho} H_{\beta \gamma \rho}\right) \Gamma_{\mu}^{\alpha \beta \gamma} \epsilon=0,
\end{aligned}
$$

for every $\epsilon$ solving (2.3). The geometric meaning of this equation is the following. Equation (2.3) defines a connection $\mathscr{D}$ on the spinor bundle by declaring that a spinor $\epsilon$ is $\mathscr{D}$-parallel if and only if it satisfies equation (2.3). Then equation (2.9) is simply the statement that $\mathscr{D}$-parallel spinors are invariant under the holonomy algebra of $\mathscr{D}$ and, in particular, are annihilated by the curvature of $\mathscr{D}$.

\subsection{Conformal symmetry superalgebras}

Let $(M, g, G, H)$ be a bosonic supersymmetric background of conformal supergravity in ten dimensions. Let $\mathfrak{C}(M, g)$ denote the Lie algebra of conformal Killing vectors on the ten-dimensional lorentzian manifold $(M, g)$. The Lie subalgebra of homothetic conformal Killing vectors will be written $\mathfrak{H}(M, g)<\mathfrak{C}(M, g)$ which contains as an ideal the Lie algebra of Killing vectors $\mathfrak{K}(M, g) \triangleleft \mathfrak{H}(M, g)$.

Now let us ascribe to $(M, g, G, H)$ a $\mathbb{Z}_{2}$-graded vector space $\mathfrak{s}=\mathfrak{s}_{\overline{0}} \oplus \mathfrak{s}_{\overline{1}}$, with even part $\mathfrak{s}_{\overline{0}} \subset \mathfrak{C}(M, g)$ and odd part $\mathfrak{s}_{\overline{1}}=\operatorname{ker} \mathscr{D}$ spanned by solutions $\epsilon$ of $(2.3)$. We would like to equip $\mathfrak{s}$ with the structure of a Lie superalgebra. The first step is to define a bracket on $\mathfrak{s}$, i.e., a skewsymmetric (in the graded sense) bilinear map $[-,-]: \mathfrak{s} \times \mathfrak{s} \rightarrow \mathfrak{s}$ such that

$$
\left[\mathfrak{s}_{\overline{0}}, \mathfrak{s}_{\overline{0}}\right] \subset \mathfrak{s}_{\overline{0}}, \quad\left[\mathfrak{s}_{\overline{0}}, \mathfrak{s}_{\overline{1}}\right] \subset \mathfrak{s}_{\overline{1}}, \quad \text { and } \quad\left[\mathfrak{s}_{\overline{1}}, \mathfrak{s}_{\overline{1}}\right] \subset \mathfrak{s}_{\overline{0}}
$$

Any such bracket on $\mathfrak{s}$ must obey the Jacobi identity in order to define a Lie superalgebra. Each graded component of the Jacobi identity is of type $[\overline{0} \overline{0} \overline{0}],[\overline{0} \overline{0} \overline{1}],[\overline{0} \overline{1} \overline{1}]$ or $[\overline{1} \overline{1} \overline{1}]$. The first three graded components can be conceptualised as follows. The $[\overline{0} \overline{0} \overline{0}]$ part says that $\mathfrak{s}_{\overline{0}}$ must be a Lie algebra with respect to $\left[\mathfrak{s}_{\overline{0}}, \mathfrak{s}_{\overline{0}}\right]$, whence $\mathfrak{s}_{\overline{0}}<\mathfrak{C}(M, g)$. The $[\overline{0} \overline{0} \overline{1}]$ part says that $\left[\mathfrak{s}_{\overline{0}}, \mathfrak{s}_{\overline{1}}\right]$ must define a representation of $\mathfrak{s}_{\overline{0}}$ on $\mathfrak{s}_{\overline{1}}$. The $[\overline{0} \overline{1} \overline{1}]$ part says that the symmetric bilinear map defined by $\left[\mathfrak{s}_{\overline{1}}, \mathfrak{s}_{\overline{1}}\right]$ must be equivariant with respect to the $\mathfrak{s}_{\overline{0}}$-action defined by $\left[\mathfrak{s}_{\overline{0}}, \mathfrak{s}_{\overline{1}}\right]$. Finally, the $[\overline{1} \overline{1} \overline{1}]$ part, being symmetric trilinear in its entries, is equivalent via polarisation to the condition

$$
[[\epsilon, \epsilon], \epsilon]=0
$$

for all $\epsilon \in \mathfrak{s}_{\overline{1}}$. If $\mathfrak{s}$ is a Lie superalgebra, notice that there exists a (possibly trivial) ideal $\mathfrak{k}_{\overline{0}}:=\left[\mathfrak{s}_{\overline{1}}, \mathfrak{s}_{\overline{1}}\right] \triangleleft \mathfrak{s}_{\overline{0}}$ and indeed $\mathfrak{k}:=\left[\mathfrak{s}_{\overline{1}}, \mathfrak{s}_{\overline{1}}\right] \oplus \mathfrak{s}_{\overline{1}} \triangleleft \mathfrak{s}$ is a Lie superalgebra ideal. 
The Kosmann-Schwarzbach Lie derivative

$$
\hat{\mathscr{L}}_{X}=\nabla_{X}+\frac{1}{4}\left(\nabla_{\mu} X_{\nu}\right) \Gamma^{\mu \nu}+\frac{1}{2} \sigma_{X} \mathbb{1},
$$

along any $X \in \mathfrak{C}(M, g)$ (i.e., $\left.\mathscr{L}_{X} g=-2 \sigma_{X} g\right)$, defines a natural conformally equivariant action of $\mathfrak{C}(M, g)$ on spinors. It is therefore tempting to define

$$
[X, \epsilon]=\hat{\mathscr{L}}_{X} \epsilon
$$

for all $X \in \mathfrak{s}_{\overline{0}}$ and $\epsilon \in \mathfrak{s}_{\overline{1}}$. However, for any $\epsilon \in \mathfrak{s}_{\overline{1}}$, one finds that $\hat{\mathscr{L}}_{X} \epsilon \in \mathfrak{s}_{\overline{1}}$ (i.e., solving (2.3)) only if

$$
\Gamma_{\mu}\left(4 \not \overrightarrow{ } \alpha_{X}+\beta_{X}\right) \epsilon+3 \beta_{X} \Gamma_{\mu} \epsilon=0,
$$

where $\alpha_{X}=G_{X}+3 \sigma_{X}$ and $\beta_{X}=\mathscr{L}_{X} H+2 \sigma_{X} H$, for all $X \in \mathfrak{s}_{\overline{0}}$. Under a Weyl transformation $(g, G, H) \mapsto\left(\Omega^{2} g, G+3 \mathrm{~d}(\ln \Omega), \Omega^{2} H\right)$ of the background, for any $X \in \mathfrak{s}_{\overline{0}}$, it follows that $\alpha_{X} \mapsto \alpha_{X}$ and $\beta_{X} \mapsto \Omega^{2} \beta_{X}$. This implies that the condition (2.14) is Weyl-invariant. If $(2.14)$ is satisfied, the bracket (2.13) solves the [ $[\overline{0} \overline{0} \overline{1}]$ Jacobi.

Now recall from above (2.7) that any $\epsilon \in \mathfrak{s}_{\overline{1}}$ has Dirac current $\xi_{\epsilon} \in \mathfrak{C}(M, g)$. Moreover, (2.7) and (2.8) are precisely the conditions $\alpha_{\xi_{\epsilon}}=0$ and $\beta_{\xi_{\epsilon}}=0$ which, if $\xi_{\epsilon} \in \mathfrak{s}_{\overline{0}}<$ $\mathfrak{C}(M, g)$, would ensure that (2.14) is satisfied. With this in mind, let us now define the $\left[\mathfrak{s}_{\overline{1}}, \mathfrak{s}_{\overline{1}}\right]$ bracket such that

$$
[\epsilon, \epsilon]=\xi_{\epsilon},
$$

for all $\epsilon \in \mathfrak{s}_{\overline{1}}$. Being symmetric bilinear in its entries, the general $\left[\mathfrak{s}_{\overline{1}}, \mathfrak{s}_{\overline{1}}\right]$ bracket follows via the polarisation $\frac{1}{2}\left(\xi_{\epsilon+\epsilon^{\prime}}-\xi_{\epsilon}-\xi_{\epsilon^{\prime}}\right)=\left[\epsilon, \epsilon^{\prime}\right]$, for any $\epsilon, \epsilon^{\prime} \in \mathfrak{s}_{\overline{1}}$. Given (2.14), it is straightforward to check that the symmetric bilinear map defined by (2.15) is indeed equivariant with

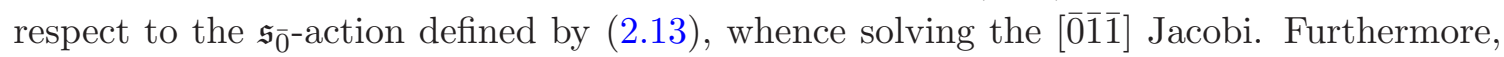
it follows using (2.5) that

$$
\left[\xi_{\epsilon}, \epsilon\right]=\hat{\mathscr{L}}_{\xi_{\epsilon}} \epsilon=0
$$

for all $\epsilon \in \mathfrak{s}_{\overline{1}}$, so the final [ $[\overline{1} \overline{1} \overline{1}]$ Jacobi is satisfied identically.

In summary, we have shown that the brackets defined by (2.13) and (2.15) equip $\mathfrak{s}$ with the structure of Lie superalgebra provided the condition (2.14) is satisfied. Any such Lie superalgebra $\mathfrak{s}$ with $\mathfrak{s}_{\overline{0}}<\mathfrak{C}(M, g)$ maximal will be referred to as the conformal symmetry superalgebra of $(M, g, G, H)$. By construction, a conformal symmetry superalgebra $\mathfrak{s}$ must have $\left[\mathfrak{s}_{\overline{1}}, \mathfrak{s}_{\overline{1}}\right] \triangleleft \mathfrak{s}_{\overline{0}}<\mathfrak{C}(M, g)$. The $\mathfrak{s}_{\overline{1}}$-generated ideal $\mathfrak{k}=\left[\mathfrak{s}_{\overline{1}}, \mathfrak{s}_{\overline{1}}\right] \oplus \mathfrak{s}_{\overline{1}}$ of a conformal symmetry superalgebra $\mathfrak{s}$ will be referred to as the conformal Killing superalgebra of $(M, g, G, H)$. It follows that every bosonic supersymmetric background of conformal supergravity in ten dimensions admits a conformal Killing superalgebra because (2.14) is identically satisfied (as a consequence of (2.7) and (2.8)) for all conformal Killing vectors in $\left[\mathfrak{s}_{\overline{1}}, \mathfrak{s}_{\overline{1}}\right]$. Of course, because the construction is manifestly Weyl-equivariant, strictly speaking a conformal symmetry superalgebra is ascribed to a conformal class of supersymmetric conformal supergravity backgrounds.

We will not attempt to obtain the general solution of (2.14) though it will be useful to describe what happens for conformal supergravity backgrounds which preserve more than half the maximal amount of supersymmetry. A simple algebraic proof was given 
in ([97], section 3.3) that any bosonic supersymmetric background of type I supergravity in ten dimensions which preserves more than half the maximal amount of supersymmetry is necessarily locally homogeneous. The same logic implies that any bosonic supersymmetric background of conformal supergravity in ten dimensions which preserves more than half the maximal amount of supersymmetry is necessarily locally conformally homogeneous. In both cases, the trick is to show that, for any given $x \in M$, the values at $x$ of all (conformal) Killing vectors $\xi_{\epsilon}$ obtained by 'squaring' supersymmetry parameters $\epsilon$ span the tangent space $T_{x} M$ (i.e., the evaluation at $x$ of the squaring map $\epsilon \mapsto \xi_{\epsilon}$ is surjective). Acting with $\bar{\epsilon} \Gamma^{\mu}$ on (2.14) implies

$$
\mathscr{L}_{\xi_{\epsilon}} \alpha_{X}=0
$$

for all $\epsilon \in \mathfrak{s}_{\overline{1}}$ and $X \in \mathfrak{s}_{\overline{0}}$. Therefore, in this case, the condition (2.17) says that $\alpha_{X}$ must be (locally) constant, for all $X \in \mathfrak{s}_{0}$. The condition (2.14) then just says that, for any vector field $Y$, the two-form $\iota_{Y} \beta_{X}$ must annihilate $\epsilon$, for all $X \in \mathfrak{s}_{\overline{0}}$ and $\epsilon \in \mathfrak{s}_{\overline{1}}$. This means that the element $\iota_{Y} \beta_{X} \in \mathfrak{s p i n}(9,1) \subset \mathrm{C} \ell(9,1)$ annihilates a linear subspace of $\Delta_{+}^{(9,1)}$ of dimension $>8$ and hence by ([98], appendix B) (see also [99], table 2) it must vanish. Thus, we have shown that demanding (2.14) for all $\epsilon \in \mathfrak{s}_{\overline{1}}$ with $\operatorname{dim} \mathfrak{s}_{\overline{1}}>8$ implies

$$
\mathrm{d} \alpha_{X}=0 \quad \text { and } \quad \beta_{X}=0,
$$

for all $X \in \mathfrak{s}_{\overline{0}}$, which then trivially implies (2.14), showing that they are equivalent.

Now consider the Weyl transformation defined above (2.4), which can be used to eliminate $G$. This maps a supersymmetric conformal supergravity background $(M, g, G, H)$ with supersymmetry parameter $\epsilon$ to another supersymmetric conformal supergravity background $\left(M, \tilde{g}=\Omega^{2} g, \tilde{G}=0, \tilde{H}=\Omega^{2} H\right)$ with supersymmetry parameter $\tilde{\epsilon}=\sqrt{\Omega} \epsilon$, where $\Omega=\mathrm{e}^{-\Phi / 3}$. If the conditions (2.18) are satisfied then

$$
\mathscr{L}_{X} \tilde{g}=-\frac{2}{3} \alpha_{X} \tilde{g} \quad \text { and } \quad \mathscr{L}_{X} \tilde{H}=0
$$

for all $X \in \mathfrak{s}_{\overline{0}}$. The first condition in (2.19) implies that every conformal Killing vector $X$ with respect to $g$ is homothetic with respect to $\tilde{g}$ (since $\alpha_{X}$ is constant), i.e., $\mathfrak{C}(M, g)=$ $\mathfrak{H}(M, \tilde{g})$. Moreover, since $\alpha_{\xi_{\epsilon}}=0$ for all $\epsilon \in \mathfrak{s}_{\overline{1}}$, every conformal Killing vector in $\left[\mathfrak{s}_{\overline{1}}, \mathfrak{s}_{\overline{1}}\right]$ is a Killing vector with respect to $\tilde{g}$. In this case, $(M, g)$ being (locally) conformally homogeneous implies that $(M, \tilde{g})$ is (locally) homogeneous.

\subsection{Maximally supersymmetric backgrounds}

Maximally supersymmetric backgrounds are such that the connection $\mathscr{D}$ defined by equation (2.3) is flat. Hence one can determine the maximally supersymmetric backgrounds of conformal supergravity in ten dimensions by solving the flatness equation which results by abstracting $\epsilon$ from equation (2.9) and solving the resulting equation for endomorphisms of the spinor bundle.

For maximally supersymmetric backgrounds of type I supergravity, the condition $G \epsilon=$ $\frac{1}{2} H \epsilon$ implies $G=0, H=0$ and (2.9) then implies that the Riemann tensor must also vanish. The only maximally supersymmetric background of type I supergravity in ten dimensions is therefore locally isometric to Minkowski space, which is Theorem 4 in [100]. 
For maximally supersymmetric backgrounds of conformal supergravity, the flatness equation derived from (2.9) implies

$$
\nabla_{\mu} H_{\nu \rho \sigma}=0, \quad H_{\mu \nu[\rho} H_{\sigma \alpha \beta]}=0, \quad \text { and } \quad H_{\mu \nu \rho} G_{\sigma}=0 .
$$

The last equation gives rise to two branches of solutions: those with $H=0$ and those with $H \neq 0$ and hence $G=0$. If $H=0$ then (2.20) are trivially satisfied and the flatness equation from (2.9) is equivalent to

$$
R_{\mu \nu \rho \sigma}=-\frac{2}{3} g_{\rho[\mu}\left(\nabla_{\nu]} G_{\sigma}+\frac{1}{3} G_{\nu]} G_{\sigma}\right)+\frac{2}{3} g_{\sigma[\mu}\left(\nabla_{\nu]} G_{\rho}+\frac{1}{3} G_{\nu]} G_{\rho}\right)+\frac{2}{9} g_{\rho[\mu} g_{\nu] \sigma} G_{\alpha} G^{\alpha} .
$$

The condition (2.21) just says that the Riemann tensor of the Weyl transformed metric $\mathrm{e}^{-2 \Phi / 3} g$ is zero. In other words, $g$ is conformally flat.

On the other hand, if $H \neq 0$, then the third condition in (2.20) implies that $G=0$ and the flatness equation derived from (2.9) is equivalent to

$$
R_{\mu \nu \rho \sigma}=\frac{1}{36}\left(3 H_{\mu \nu}{ }^{\alpha} H_{\rho \sigma \alpha}+g_{\rho[\mu} H_{\nu]}^{\alpha \beta} H_{\sigma \alpha \beta}-g_{\sigma[\mu} H_{\nu]}^{\alpha \beta} H_{\rho \alpha \beta}-\frac{1}{3} g_{\rho[\mu} g_{\nu] \sigma} H^{\alpha \beta \gamma} H_{\alpha \beta \gamma}\right),
$$

together with the first two conditions in (2.20). The first of those conditions says that $H$ is parallel with respect to the Levi-Civita connection $\nabla$ and, by equation (2.22), so is the Riemann tensor of $g$. In other words, the background must be locally isometric to a lorentzian symmetric space. Now we shall classify maximally supersymmetric backgrounds of conformal supergravity with $G=0$, making use of several key techniques developed in [100].

The second condition in (2.20), written in a more invariant way, is

$$
\iota_{X} \iota_{Y} H \wedge H=0
$$

for all vector fields $X, Y$. This is none other than the family of Plücker quadrics for $H$ (see, e.g., [101], Chapter 1), which is equivalent to $H$ being decomposable; that is, $H=\alpha \wedge \beta \wedge \gamma$, for one-forms $\alpha, \beta, \gamma$. Any background of interest is therefore locally isometric to a tendimensional lorentzian symmetric space $M$ equipped with a parallel decomposable threeform $H$. These conditions are quite restrictive and solutions are distinguished according to whether the constant $\|H\|^{2}:=\frac{1}{6} H_{\mu \nu \rho} H^{\mu \nu \rho}$ is positive, negative or zero. The geometric meaning of this constant has to do with the metric nature of the tangent 3-planes which $H$ defines: they can be either euclidean, lorentzian or degenerate, according to whether $\|H\|^{2}$ is positive, negative or zero, respectively. From (2.22), it follows that the constant scalar curvature of $g$ is $R=-\frac{1}{2}\|H\|^{2}$. The maximally supersymmetric backgrounds are summarised below (with the scalar curvature of each $A d S$ and $S$ factor denoted in parenthesis).

- If $R>0, M=A d S_{3}\left(-\frac{4}{3} R\right) \times S^{7}\left(\frac{7}{3} R\right)$ with $H=\sqrt{2 R} \operatorname{vol}_{A d S_{3}}$.

- If $R<0, M=A d S_{7}\left(\frac{7}{3} R\right) \times S^{3}\left(-\frac{4}{3} R\right)$ with $H=\sqrt{-2 R} \operatorname{vol}_{S^{3}}$.

- If $R=0, M=C W_{10}(A)$ with $A=-\frac{\mu^{2}}{36} \operatorname{diag}(4,4,1,1,1,1,1,1)$ and $H=\mu \mathrm{d} x^{-} \wedge$ $\mathrm{d} x^{1} \wedge \mathrm{d} x^{2}$. 
The background $C W_{10}(A)$ denotes a ten-dimensional Cahen-Wallach lorentzian symmetric space with metric

$$
g=2 \mathrm{~d} x^{+} \mathrm{d} x^{-}+\left(\sum_{a, b=1}^{8} A_{a b} x^{a} x^{b}\right)\left(\mathrm{d} x^{-}\right)^{2}+\sum_{a=1}^{8}\left(\mathrm{~d} x^{a}\right)^{2},
$$

in terms of local coordinates $\left(x^{ \pm}, x^{a}\right)$. For a general constant symmetric matrix $A=\left(A_{a b}\right)$, it follows that $g$ is conformally flat only if $A$ is proportional to the identity matrix. Clearly this is not the case for the particular $A$ which defines the maximally supersymmetric background in the third item above (unless $\mu=0$, in which case $C W_{10}(0)=\mathbb{R}^{9,1}$ ). Moreover, the maximally supersymmetric backgrounds with $R \neq 0$ in the first two items above are not conformally flat since, in each case, the constant sectional curvatures of the $\operatorname{AdS}$ and $S$ factors are not equal and opposite (e.g., see (1.167) in [102]).

It follows from ([103], section 4) that the Freund-Rubin backgrounds $A d S_{3} \times S^{7}$ and $A d S_{7} \times S^{3}$ found above have two distinct plane-wave (or Penrose-Güven) limits up to local isometry. If the geodetic vector of the null geodesic along which we take the limit is tangent to the anti-de Sitter space, then the limit is flat, whereas if the geodetic vector has a nonzero component tangent to the sphere, the limit is isometric to the Cahen-Wallach background we found above. Indeed, the ratio $(=4$, in this case) between the two eigenvalues of the symmetric matrix $A$ defining the Cahen-Wallach metric is the square of the ratio $(=2$, in this case) of the radii of curvature of the 3 - and 7-dimensional factors in the Freund-Rubin geometry. This gives another proof that the Freund-Rubin backgrounds are not conformally flat, since conformal flatness is a hereditary property under the plane-wave limit ([103], section 3.2$)$, but the $C W_{10}(A)$ geometry above is not conformally flat for $\mu \neq 0$.

\subsubsection{Conformal symmetry superalgebras}

Let us now investigate how the construction of conformal symmetry superalgebras in section 2.3 plays out for the maximally supersymmetric conformal supergravity backgrounds we have just classified.

For the maximally supersymmetric background with $H=0,(M, \tilde{g})$ is locally isometric to $\mathbb{R}^{9,1}$. The supersymmetry condition (2.4) implies $\mathfrak{s}_{\overline{1}} \cong \Delta_{+}^{(9,1)}$ on $\mathbb{R}^{9,1}$. Surjectivity of the squaring map then implies $\left[\mathfrak{s}_{\overline{1}}, \mathfrak{s}_{\overline{1}}\right] \cong \mathbb{R}^{9,1}$. For any $\epsilon \in \Delta_{+}^{(9,1)}, \hat{\mathscr{L}}_{X} \epsilon \in \Delta_{+}^{(9,1)}$ only if the conformal Killing vector $X$ does not involve a special conformal transformation in $\mathfrak{C}\left(\mathbb{R}^{9,1}\right) \cong$ $\mathfrak{s o}(10,2)$. This is just as expected from (2.18), so that the associated conformal factor $\sigma_{X}$ is constant. Thus, we must take $\mathfrak{s}_{\overline{0}}=\mathfrak{H}\left(\mathbb{R}^{9,1}\right)<\mathfrak{C}\left(\mathbb{R}^{9,1}\right)$, which consists of the obvious Poincaré transformations generated by $\mathfrak{K}\left(\mathbb{R}^{9,1}\right) \cong \mathfrak{s o}(9,1) \ltimes \mathbb{R}^{9,1}$ plus dilatation generated by a proper homothetic conformal Killing vector $\theta$. The Lie superalgebra obtained by restricting to $\mathfrak{K}\left(\mathbb{R}^{9,1}\right) \triangleleft \mathfrak{H}\left(\mathbb{R}^{9,1}\right)$ is isomorphic to the Poincaré superalgebra in ten dimensions. The conformal symmetry superalgebra $\mathfrak{s}$ merely appends $\theta$ to this Poincaré superalgebra, with the additional bracket $[\theta, \epsilon]=\frac{1}{2} \epsilon$, for all $\epsilon \in \Delta_{+}^{(9,1)}$ (which implies $\left[\theta, \xi_{\epsilon}\right]=\xi_{\epsilon}$ ).

For all three maximally supersymmetric backgrounds with $H \neq 0, G=0$ so $\mathfrak{C}(M, g)=\mathfrak{H}(M, g)$ because $\alpha_{X}=3 \sigma_{X}$ is constant, for all $X \in \mathfrak{C}(M, g)$. Given any $X, Y \in \mathfrak{H}(M, g)$ with $\sigma_{X} \neq 0$, then $Y-\frac{\sigma_{Y}}{\sigma_{X}} X \in \mathfrak{K}(M, g)$. Hence, either $\mathfrak{H}(M, g)=\mathfrak{K}(M, g)$ 
or $\operatorname{dim}(\mathfrak{H}(M, g) / \mathfrak{K}(M, g))=1$. For the two maximally supersymmetric backgrounds with constant scalar curvature $R \neq 0$, a quick calculation reveals that the scalar normsquared of the Weyl tensor $W$ of $g$ is $\|W\|^{2}=\frac{7}{36} R^{2}$. Recall that the Weyl tensor obeys $\mathscr{L}_{X} W=-2 \sigma_{X} W$, for all $X \in \mathfrak{C}(M, g)$, so $\mathscr{L}_{X}\|W\|^{2}=4 \sigma_{X}\|W\|^{2}$. Hence, because in this case $\|W\|^{2}$ is a non-zero constant, it follows that $\mathfrak{H}(M, g)=\mathfrak{K}(M, g)$. The third maximally supersymmetric background with $R=0$ does admit a proper homothetic conformal Killing vector so $\operatorname{dim}(\mathfrak{H}(M, g) / \mathfrak{K}(M, g))=1$.

\subsection{2 $\mathfrak{s}\left(A d S_{3} \times S^{7}\right)$ and $\mathfrak{s}\left(A d S_{7} \times S^{3}\right)$}

The preceding discussion has established that the only conformal Killing vectors for these two geometries are Killing vectors. Moreover, it is not difficult to prove that all such Killing vectors correspond to Killing vectors on the individual $A d S$ and $S$ factors.

The supersymmetry condition (2.4) reduces to a pair of Killing spinor equations on the individual $A d S$ and $S$ factors. In our conventions, a spinor $\psi$ on a lorentzian/riemannian spin manifold $M$ is Killing if, for any vector field $X$ on $M$, it obeys $\nabla_{X} \psi= \pm \frac{\kappa}{2} X \psi$, for some real/imaginary constant $\kappa$. In the case at hand, the Killing constants are given by $\kappa_{A d S_{3}}=i \kappa_{S^{3}}=\frac{1}{3} \sqrt{2|R|}$ and $\kappa_{A d S_{7}}=i \kappa_{S^{7}}=\frac{1}{6} \sqrt{2|R|}$.

Both $A d S_{m}$ and $S^{n}$ can be described via the canonical quadric embedding in (an open subset of) $\mathbb{R}^{m-1,2}$ and $\mathbb{R}^{n+1}$ respectively. Conversely, the flat metrics on both $\mathbb{R}^{m-1,2}$ and $\mathbb{R}^{n+1}$ can be written as (lorentzian and riemannian) cone metrics whose bases form the respective $A d S_{m}$ and $S^{n}$ geometries. This cone construction is particularly useful in describing Killing vectors and Killing spinors on these geometries (see [104] for a review in a similar context). Every Killing vector on the base lifts to a constant two-form on the cone and vice versa. Thus $\mathfrak{K}\left(A d S_{m}\right) \cong \mathfrak{s o}(m-1,2)\left(\cong \wedge^{2} \mathbb{R}^{m-1,2}\right.$ as a vector space $)$ and $\mathfrak{K}\left(S^{n}\right) \cong \mathfrak{s o}(n+1)\left(\cong \wedge^{2} \mathbb{R}^{n+1}\right.$ as a vector space $)$. Every Killing spinor on the base lifts to a constant spinor on the cone and vice versa. More precisely, if both $m$ and $n$ are odd, there is a bijection between Killing spinors on the base and constant chiral spinors on the cone. The Kosmann-Schwarzbach Lie derivative of a Killing spinor along a Killing vector on the base lifts to the obvious Clifford action of a constant two-form on a constant spinor on the cone.

The cleanest way to discuss the explicit structure of $\mathfrak{s}\left(A d S_{3} \times S^{7}\right)$ and $\mathfrak{s}\left(A d S_{7} \times S^{3}\right)$ is as particular real forms of the same complex Lie superalgebra $\mathfrak{s}^{\mathbb{C}}$. The even part of $\mathfrak{s}^{\mathbb{C}}$ is $\mathfrak{s}_{\overline{0}}^{\mathbb{C}}=\mathfrak{s o}_{4}(\mathbb{C}) \oplus \mathfrak{s o}_{8}(\mathbb{C})$. The odd part of $\mathfrak{s}^{\mathbb{C}}$ is $\mathfrak{s}_{\overline{1}}^{\mathbb{C}}=\Delta_{+}^{(4, \mathbb{C})} \otimes \Delta_{+}^{(8, \mathbb{C})}$, where $\Delta_{+}^{(4, \mathbb{C})} \cong \mathbb{C}^{2}$ and $\Delta_{+}^{(8, \mathbb{C})} \cong \mathbb{C}^{8}$ denote the chiral spinor representations of the respective $\mathfrak{s o}_{4}(\mathbb{C})$ and $\mathfrak{s o}_{8}(\mathbb{C})$ factors in $\mathfrak{s}_{\overline{0}}^{\mathbb{C}}$. Let $\langle-,-\rangle$ denote the unique (up to scale) $\mathfrak{s o}_{4}(\mathbb{C})$-invariant skewsymmetric complex bilinear form on $\Delta_{+}^{(4, \mathbb{C})}$ and let $(-,-)$ denote the unique (up to scale) $\mathfrak{s o}_{8}(\mathbb{C}$ )invariant symmetric complex bilinear form on $\Delta_{+}^{(8, \mathbb{C})}$.

Now fix a basis $\left(L_{A B}=-L_{B A}, M_{I J}=-M_{J I}\right)$ for $\mathfrak{s}_{0}^{\mathbb{C}}$, where $A, B=1,2,3,4$ and $I, J=1, \ldots, 8$. The brackets for $\mathfrak{s}^{\mathbb{C}}$ are as follows

$$
\begin{aligned}
{\left[L_{A B}, L_{C D}\right] } & =-\delta_{A C} L_{B D}+\delta_{B C} L_{A D}+\delta_{A D} L_{B C}-\delta_{B D} L_{A C}, \\
{\left[M_{I J}, M_{K L}\right] } & =-\delta_{I K} M_{J L}+\delta_{J K} M_{I L}+\delta_{I L} M_{J K}-\delta_{J L} M_{I K}, \\
{\left[L_{A B}, \psi \otimes \varphi\right] } & =\frac{1}{2} \gamma_{A B} \psi \otimes \varphi
\end{aligned}
$$




\begin{tabular}{|c|c|c|c|c|}
\hline$M$ & $\mathfrak{s}_{\overline{0}}$ & $\mathfrak{s}_{\overline{1}}$ & type & $\mathfrak{s}$ \\
\hline$A d S_{3} \times S^{7}$ & $\mathfrak{s o}(2,2) \oplus \mathfrak{s o}(8)$ & $\Delta_{+}^{(2,2)} \otimes \Delta_{+}^{(8)}$ & $\mathbb{R}$ & $\mathfrak{s l}_{2}(\mathbb{R}) \oplus \mathfrak{o s p}(8 \mid 2)$ \\
\hline$A d S_{7} \times S^{3}$ & $\mathfrak{s o}(6,2) \oplus \mathfrak{s o}(4)$ & {$\left[\Delta_{+}^{(6,2)} \otimes \Delta_{+}^{(4)}\right]$} & $\mathbb{H}$ & $\mathfrak{o} \mathfrak{s p}(6,2 \mid 1) \oplus \mathfrak{s p}(1)$ \\
\hline
\end{tabular}

Table 1. Data for admissible real forms of $\mathfrak{s}^{\mathbb{C}} \cong \mathfrak{s p}_{1}(\mathbb{C}) \oplus \mathfrak{o s p} \mathfrak{p}_{8 \mid 1}(\mathbb{C})$.

$$
\begin{aligned}
{\left[M_{I J}, \psi \otimes \varphi\right] } & =\frac{1}{2} \psi \otimes \gamma_{I J} \varphi \\
{\left[\psi \otimes \varphi, \psi^{\prime} \otimes \varphi^{\prime}\right] } & =\left\langle\psi, \gamma^{A B} \psi^{\prime}\right\rangle\left(\varphi, \varphi^{\prime}\right) L_{A B}-\frac{1}{2}\left\langle\psi, \psi^{\prime}\right\rangle\left(\varphi, \gamma^{I J} \varphi^{\prime}\right) M_{I J},
\end{aligned}
$$

for all $\psi, \psi^{\prime} \in \Delta_{+}^{(4, \mathbb{C})}$ and $\varphi, \varphi^{\prime} \in \Delta_{+}^{(8, \mathbb{C})}$, where $\left\{\gamma_{A}\right\}$ generate $\mathbb{C} \ell(4)$ and $\left\{\gamma_{I}\right\}$ generate $\mathbb{C} \ell(8)$. It is a straightforward exercise to check that (2.25) obey the graded Jacobi identities; although the $[\overline{1} \overline{1} \overline{1}]$ component requires use of the following identities,

$$
\gamma^{A B} \psi\left\langle\gamma_{A B} \psi,-\right\rangle=-4 \psi\langle\psi,-\rangle
$$

and

$$
\gamma^{I J} \varphi\left(\gamma_{I J} \varphi,-\right)=-8 \varphi(\varphi,-)+8(\varphi, \varphi) \mathbb{1},
$$

which hold for all $\psi \in \Delta_{+}^{(4, \mathbb{C})}$ and $\varphi \in \Delta_{+}^{(8, \mathbb{C})}$.

As a vector space, $\mathfrak{s o}_{4}(\mathbb{C}) \cong \wedge^{2} \mathbb{C}^{4} \cong \wedge_{+}^{2} \mathbb{C}^{4} \oplus \wedge_{-}^{2} \mathbb{C}^{4}$, in terms of the vector spaces $\wedge_{ \pm}^{2} \mathbb{C}^{4}$ of (anti)self-dual two-forms on $\mathbb{C}^{4}$ which span each $\mathfrak{s p}_{1}(\mathbb{C})$ factor in $\mathfrak{s o}_{4}(\mathbb{C}) \cong \mathfrak{s p}_{1}(\mathbb{C}) \oplus \mathfrak{s p}_{1}(\mathbb{C})$. Let $\varepsilon \in \wedge^{4} \mathbb{C}^{4}$ with $\varepsilon_{1234}=1$ and let $\gamma=-\gamma_{1234}$ define the chirality matrix for $\mathbb{C} \ell(4)$. It follows that $\gamma_{A B} \gamma=\frac{1}{2} \varepsilon_{A B C D} \gamma^{C D}$, so any $\psi \in \Delta_{+}^{(4, \mathbb{C})}$ defines a self-dual two-form $\left\langle\psi, \gamma_{A B} \psi\right\rangle$. This implies that the bracket defined by $(2.25)$ of the $\mathfrak{s p}_{1}(\mathbb{C})<\mathfrak{s o}_{4}(\mathbb{C})$ spanned by $\wedge_{-}^{2} \mathbb{C}^{4}$ with every other element in $\mathfrak{s}^{\mathbb{C}}$ is zero. The action of the other $\mathfrak{s p}_{1}(\mathbb{C})<\mathfrak{s o}_{4}(\mathbb{C})$ (spanned by $\left.\wedge_{+}^{2} \mathbb{C}^{4}\right)$ on $\Delta_{+}^{(4, \mathbb{C})}$ just corresponds to the defining representation $\Delta^{\mathbb{C}}$ of this $\mathfrak{s p}_{1}(\mathbb{C})$. Excluding the decoupled $\mathfrak{s p}_{1}(\mathbb{C})$ factor from $\mathfrak{s}^{\mathbb{C}}$ leaves a simple complex Lie superalgebra that is isomorphic to $\mathfrak{o s p}_{8 \mid 1}(\mathbb{C})$ (a.k.a. $D(4,1)$ in the Kac classification [105]), with even part $\mathfrak{s o}_{8}(\mathbb{C}) \oplus \mathfrak{s p}_{1}(\mathbb{C})$ and odd part $\Delta_{+}^{(8, \mathbb{C})} \otimes \Delta^{\mathbb{C}}$. Thus, $\mathfrak{s}^{\mathbb{C}} \cong \mathfrak{s p}_{1}(\mathbb{C}) \oplus \mathfrak{o s p}_{8 \mid 1}(\mathbb{C})$.

The real forms of all complex classical Lie superalgebras in [105] were classified in [106]. Up to isomorphism, the real forms of a given complex classical Lie superalgebra are uniquely determined by the real forms of the complex reductive Lie algebra which constitutes its even part. The even part of $\mathfrak{s}^{\mathbb{C}}$ is $\mathfrak{s}_{0}^{\mathbb{C}}=\mathfrak{s o}_{4}(\mathbb{C}) \oplus \mathfrak{s o}_{8}(\mathbb{C})$ which admits many non-isomorphic real forms. However, of these real forms, only $\mathfrak{s o}(2,2) \oplus \mathfrak{s o}(8)$ and $\mathfrak{s o}(6,2) \oplus \mathfrak{s o}(4)$ are isomorphic to the Lie algebra of isometries of $A d S_{3} \times S^{7}$ and $A d S_{7} \times S^{3}$, respectively. It is then straightforward to deduce the associated real forms which describe their conformal symmetry superalgebras $\mathfrak{s}$. The pertinent data is summarised in table 1 .

We have opted for the more common physics notation to write the real form $\mathfrak{o s p}(8 \mid 2)$ rather than its perhaps more logical alias $\mathfrak{o s p}_{8 \mid 1}(\mathbb{R})$. The notation is that $\Delta_{+}^{(p, q)}$ denotes the positive-chirality spinor representation of $\mathfrak{s o}(p, q)$ when $p+q$ is even. As vector spaces, $\Delta_{+}^{(2,2)} \cong \mathbb{R}^{2}, \Delta_{+}^{(8)} \cong \mathbb{R}^{8}, \Delta_{+}^{(6,2)} \cong \mathbb{H}^{4}$ and $\Delta_{+}^{(4)} \cong \mathbb{H}$. Given a pair of quaternionic representations $W_{1}$ and $W_{2}$, which we think of as complex representations equipped 
with invariant quaternionic structures $J_{1}$ and $J_{2}$, their tensor product $J_{1} \otimes J_{2}$ defines a real structure on $W_{1} \otimes W_{2}$, where the tensor product is over $\mathbb{C}$. This means that $W_{1} \otimes W_{2} \cong \mathbb{C} \otimes_{\mathbb{R}}\left[W_{1} \otimes W_{2}\right]$, where $\left[W_{1} \otimes W_{2}\right]$ is a real representation which can be identified with the subspace of real elements (i.e., fixed points of the real structure) in $W_{1} \otimes W_{2}$. Note that $\mathfrak{s o}(2,2) \cong \mathfrak{s l}_{2}(\mathbb{R}) \oplus \mathfrak{s l}_{2}(\mathbb{R})$ with $\Delta_{+}^{(2,2)} \cong \Delta \otimes \mathbb{R}$, in terms of the defining representation $\Delta$ of $\mathfrak{s l}_{2}(\mathbb{R})$, while $\mathfrak{s o}(4) \cong \mathfrak{s p}(1) \oplus \mathfrak{s p}(1)$ with $\Delta_{+}^{(4)} \cong \Delta^{\prime} \otimes \mathbb{R}$, in terms of the defining representation $\Delta^{\prime}$ of $\mathfrak{s p}(1)$, where we use $\mathbb{R}$ for the trivial real representation.

\subsection{3 $\mathfrak{s}\left(C W_{10}(A)\right)$}

To describe the conformal Killing vectors of the Cahen-Wallach geometry (2.24) with $A=$ $-\frac{\mu^{2}}{36} \operatorname{diag}(4,4,1,1,1,1,1,1)$, it is convenient to partition the indices $a, b, \ldots$, which take values in $\{1, \ldots, 8\}$, into $\alpha, \beta, \ldots \in\{1,2\}$ and $i, j, \ldots \in\{3, \ldots, 8\}$.

A basis of Killing vectors for this geometry is given by

$$
\begin{aligned}
\xi & =\partial_{+} & q_{\alpha} & =\frac{3}{\mu} \sin \left(\frac{\mu}{3} x^{-}\right) \partial_{\alpha}-x^{\alpha} \cos \left(\frac{\mu}{3} x^{-}\right) \partial_{+} \\
\zeta & =\partial_{-} & q_{i} & =\frac{6}{\mu} \sin \left(\frac{\mu}{6} x^{-}\right) \partial_{i}-x^{i} \cos \left(\frac{\mu}{6} x^{-}\right) \partial_{+} \\
J & =x^{1} \partial_{2}-x^{2} \partial_{1} & p_{\alpha} & =\cos \left(\frac{\mu}{3} x^{-}\right) \partial_{\alpha}+\frac{\mu}{3} x^{\alpha} \sin \left(\frac{\mu}{3} x^{-}\right) \partial_{+} \\
M_{i j} & =x^{i} \partial_{j}-x^{j} \partial_{i} & p_{i} & =\cos \left(\frac{\mu}{6} x^{-}\right) \partial_{i}+\frac{\mu}{6} x^{i} \sin \left(\frac{\mu}{6} x^{-}\right) \partial_{+} .
\end{aligned}
$$

Their non-vanishing Lie brackets are as follows

$$
\begin{aligned}
& {\left[\zeta, q_{\alpha}\right]=p_{\alpha}} \\
& {\left[\zeta, q_{i}\right]=p_{i}} \\
& {\left[\zeta, p_{\alpha}\right]=-\frac{\mu^{2}}{9} q_{\alpha}} \\
& {\left[J, q_{1}\right]=-q_{2}} \\
& {\left[q_{i}, p_{j}\right]=\delta_{i j} \xi} \\
& {\left[J, q_{2}\right]=q_{1}} \\
& {\left[q_{\alpha}, p_{\beta}\right]=\delta_{\alpha \beta} \xi} \\
& {\left[\zeta, p_{i}\right]=-\frac{\mu^{2}}{36} q_{i}} \\
& {\left[J, p_{1}\right]=-p_{2}} \\
& {\left[M_{i j}, q_{k}\right]=-\delta_{i k} q_{j}+\delta_{j k} q_{i}} \\
& {\left[J, p_{2}\right]=p_{1}} \\
& {\left[M_{i j}, p_{k}\right]=-\delta_{i k} p_{j}+\delta_{j k} p_{i}}
\end{aligned}
$$

in addition to

$$
\left[M_{i j}, M_{k l}\right]=-\delta_{i k} M_{j l}+\delta_{j k} M_{i l}+\delta_{i l} M_{j k}-\delta_{j l} M_{i k} .
$$

The Killing vectors $(\xi, q, p)$ are generic for plane wave geometries and we see from (2.29) that they form a 17-dimensional Lie subalgebra isomorphic to the Heisenberg algebra $\mathfrak{h e i s _ { 8 }}(\mathbb{R})$. The Killing vectors $(J, M)$ span the Lie subalgebra $\mathfrak{s o}(2) \oplus \mathfrak{s o}(6)<\mathfrak{s o}(8)$ which stabilises $A$.

In total, notice that $\operatorname{dim} \mathfrak{K}\left(C W_{10}(A)\right)=34=\operatorname{dim} \mathfrak{K}\left(A d S_{3} \times S^{7}\right)=\operatorname{dim} \mathfrak{K}\left(A d S_{7} \times S^{3}\right)$. However, $C W_{10}(A)$ admits an additional homothetic conformal Killing vector

$$
\theta=2 x^{+} \partial_{+}+x^{a} \partial_{a}
$$

normalised such that $\sigma_{\theta}=-1$. Its non-vanishing Lie brackets with $\mathfrak{K}\left(C W_{10}(A)\right)$ are

$$
[\xi, \theta]=2 \xi, \quad\left[q_{a}, \theta\right]=q_{a} \quad \text { and } \quad\left[p_{a}, \theta\right]=p_{a}
$$


Thus we have obtained $\mathfrak{s}_{\overline{0}}\left(C W_{10}(A)\right)$, defined with respect to the basis $(\xi, q, p, \zeta, J, M, \theta) \in$ $\mathfrak{H}\left(C W_{10}(A)\right)$, subject to Lie brackets $(2.29)$ and (2.32).

The general solution of $(2.4)$ on $C W_{10}(A)$ yields a supersymmetry parameter of the form

$$
\epsilon=\exp \left(\frac{\mu}{4} x^{-} \boldsymbol{I}\right) \eta_{+}+\frac{1}{2}\left(\Gamma_{-}+\frac{\mu}{3}\left(x^{\alpha} \Gamma_{\alpha}-\frac{1}{2} x^{i} \Gamma_{i}\right) \boldsymbol{I}\right) \exp \left(\frac{\mu}{12} x^{-} \boldsymbol{I}\right) \eta_{-},
$$

in terms of $\boldsymbol{I}=\Gamma_{12}$ and any pair of constant spinors $\eta_{ \pm} \in \Delta_{ \pm}^{(9,1)}$ with $\Gamma_{+} \eta_{ \pm}=0$. It is perhaps worth noting that a spinor of the form $(2.33)$ on $C W_{10}(A)$ cannot be parallel with respect to the Levi-Civita connection $\nabla$ unless it is identically zero because any $\nabla$-parallel spinor $\psi$ on $C W_{10}(A)$ is necessarily constant with $\Gamma_{+} \psi=0$.

Now let us adopt the shorthand notation $\epsilon=\Psi\left(\eta_{+}, \eta_{-}\right)$for any supersymmetry parameter of the form (2.33). Substituting (2.28), (2.31) and (2.33) into the KosmannSchwarzbach Lie derivative (2.12) yields the following non-vanishing even-odd brackets for $\mathfrak{s}\left(C W_{10}(A)\right)$

$$
\begin{aligned}
{\left[\zeta, \Psi\left(\eta_{+}, \eta_{-}\right)\right] } & =\frac{\mu}{4} \Psi\left(\boldsymbol{I} \eta_{+}, \frac{1}{3} \boldsymbol{I} \eta_{-}\right) & {\left[\theta, \Psi\left(\eta_{+}, \eta_{-}\right)\right] } & =-\Psi\left(\eta_{+}, 0\right) \\
{\left[J, \Psi\left(\eta_{+}, \eta_{-}\right)\right] } & =\frac{1}{2} \Psi\left(\boldsymbol{I} \eta_{+}, \boldsymbol{I} \eta_{-}\right) & {\left[M_{i j}, \Psi\left(\eta_{+}, \eta_{-}\right)\right] } & =\frac{1}{2} \Psi\left(\Gamma_{i j} \eta_{+}, \Gamma_{i j} \eta_{-}\right) \\
{\left[q_{\alpha}, \Psi\left(\eta_{+}, \eta_{-}\right)\right] } & =-\frac{1}{2} \Psi\left(\Gamma_{\alpha} \eta_{-}, 0\right) & {\left[q_{i}, \Psi\left(\eta_{+}, \eta_{-}\right)\right] } & =-\frac{1}{2} \Psi\left(\Gamma_{i} \eta_{-}, 0\right) \\
{\left[p_{\alpha}, \Psi\left(\eta_{+}, \eta_{-}\right)\right] } & =\frac{\mu}{6} \Psi\left(\Gamma_{\alpha} \boldsymbol{I} \eta_{-}, 0\right) & {\left[p_{i}, \Psi\left(\eta_{+}, \eta_{-}\right)\right] } & =-\frac{\mu}{12} \Psi\left(\Gamma_{i} \boldsymbol{I} \eta_{-}, 0\right) .
\end{aligned}
$$

Notice, in particular, that $\xi$ acts trivially on the Killing spinors.

Finally, substituting (2.33) into the squaring map $\epsilon \mapsto \xi_{\epsilon}$ gives the odd-odd bracket

$$
\begin{aligned}
{[\epsilon, \epsilon]=} & \left(\bar{\eta}_{+} \Gamma_{-} \eta_{+}\right) \xi-\frac{1}{2}\left(\bar{\eta}_{-} \Gamma_{-} \eta_{-}\right)\left(\zeta+\frac{\mu}{3} J\right)-\frac{\mu}{24}\left(\bar{\eta}_{-} \Gamma_{-} \Gamma^{i j} \boldsymbol{I} \eta_{-}\right) M_{i j} \\
& -\frac{\mu}{3}\left(\bar{\eta}_{+} \Gamma_{-} \Gamma^{\alpha} \boldsymbol{I} \eta_{-}\right) q_{\alpha}+\frac{\mu}{6}\left(\bar{\eta}_{+} \Gamma_{-} \Gamma^{i} \boldsymbol{I} \eta_{-}\right) q_{i}-\left(\bar{\eta}_{+} \Gamma_{-} \Gamma^{\alpha} \eta_{-}\right) p_{\alpha}-\left(\bar{\eta}_{+} \Gamma_{-} \Gamma^{i} \eta_{-}\right) p_{i} .
\end{aligned}
$$

Thus we have obtained $\mathfrak{s}\left(C W_{10}(A)\right)$ and it is a simple matter to confirm that the brackets defined by equations (2.29), (2.30), (2.32), (2.34) and (2.35) indeed obey the Jacobi identities. For any $X \in \mathfrak{H}\left(C W_{10}(A)\right), \alpha_{X}=3 \sigma_{X}$ is obviously constant and one can check that $\beta_{X}=0$, as expected from (2.18), because the three-form $H=\mu \mathrm{d} x^{-} \wedge \mathrm{d} x^{1} \wedge \mathrm{d} x^{2}$ obeys $\mathscr{L}_{X} H=-2 \sigma_{X} H$.

\subsubsection{Contractions}

As we have seen, the Cahen-Wallach background is the plane-wave limit of the FreundRubin backgrounds. Therefore we might expect, based on what happens in ten- and eleven-dimensional Poincaré supergravities [103, 107], that $\mathfrak{s}\left(C W_{10}(A)\right)$ is a contraction (in the sense of Inönü-Wigner) of $\mathfrak{s}\left(A d S_{3} \times S^{7}\right)$ and $\mathfrak{s}\left(A d S_{7} \times S^{3}\right)$. Indeed, it was precisely that observation in [108] which led to the identification of the maximally supersymmetric plane-wave solutions of eleven-dimensional and IIB supergravities as plane-wave limits of 
the corresponding Freund-Rubin solutions in [109]. We will give the details only for the $A d S_{3} \times S^{7}$ Freund-Rubin background, and leave the similar calculation for $A d S_{7} \times S^{3}$ to the imagination.

The contraction is easiest to describe in the following basis. Let $\mathfrak{s}_{\overline{0}}=\mathfrak{s o}(2,2) \oplus \mathfrak{s o}(8)$ be the even part of $\mathfrak{s}\left(A d S_{3} \times S^{7}\right)$. We will choose a basis $\left(P_{\mu}, L_{\mu \nu}\right)$ for $\mathfrak{s o}(2,2)$ and $\left(P_{m}, M_{m n}\right)$ for $\mathfrak{s o}(8)$, where $\mu, \nu=0,1,2$ and $m, n=3, \ldots, 9$. The Lie brackets are given by

$$
\begin{aligned}
{\left[P_{\mu}, P_{\nu}\right] } & =4 L_{\mu \nu} & {\left[P_{m}, P_{n}\right] } & =-M_{m n} \\
{\left[L_{\mu \nu}, P_{\rho}\right] } & =-\eta_{\mu \rho} P_{\nu}+\eta_{\nu \rho} P_{\mu} & {\left[M_{m n}, P_{p}\right] } & =-\delta_{m p} P_{n}+\delta_{n p} P_{m}
\end{aligned}
$$

and

$$
\begin{aligned}
{\left[L_{\mu \nu}, L_{\rho \sigma}\right] } & =-\eta_{\mu \rho} L_{\nu \sigma}+\eta_{\nu \rho} L_{\mu \sigma}+\eta_{\mu \sigma} L_{\nu \rho}-\eta_{\nu \sigma} L_{\mu \rho} \\
{\left[M_{m n}, M_{p q}\right] } & =-\delta_{m p} M_{n q}+\delta_{n p} M_{m q}+\delta_{m q} M_{n p}-\delta_{n q} M_{m p}
\end{aligned}
$$

where $\eta=\operatorname{diag}(-1,+1,+1)$. Let $\Psi: \Delta_{+}^{(9,1)} \rightarrow \mathfrak{s}_{\overline{1}}$ be a vector space isomorphism. The even-odd brackets of the conformal symmetry superalgebra of $A d S_{3} \times S^{7}$ are given by

$$
\begin{aligned}
& {\left[L_{\mu \nu}, \Psi(\varepsilon)\right]=\Psi\left(\frac{1}{2} \Gamma_{\mu \nu} \varepsilon\right) \quad \text { and } \quad\left[M_{m n}, \Psi(\varepsilon)\right]=\Psi\left(\frac{1}{2} \Gamma_{m n} \varepsilon\right),} \\
& {\left[P_{\mu}, \Psi(\varepsilon)\right]=\Psi\left(\Gamma_{\mu} \nu \varepsilon\right) \quad \text { and } \quad\left[P_{m}, \Psi(\varepsilon)\right]=-\frac{1}{2} \Psi\left(\Gamma_{m} \nu \varepsilon\right),}
\end{aligned}
$$

in terms of the $\mathrm{C} \ell(9,1)$ gamma matrices and where $\varepsilon \in \Delta_{+}^{(9,1)}$ and $\nu=\Gamma_{012}$. Finally, the odd-odd brackets are given by

$$
[\Psi(\varepsilon), \Psi(\varepsilon)]=\left(\bar{\varepsilon} \Gamma^{\mu} \varepsilon\right) P_{\mu}+\left(\bar{\varepsilon} \Gamma^{m} \varepsilon\right) P_{m}-\left(\bar{\varepsilon} \Gamma^{\mu \nu} \nu \varepsilon\right) L_{\mu \nu}+\frac{1}{2}\left(\bar{\varepsilon} \Gamma^{m n} \nu \varepsilon\right) M_{m n} .
$$

We now decompose $\varepsilon=\varepsilon_{+}+\varepsilon_{-}$, with $\varepsilon_{ \pm} \in \operatorname{ker} \Gamma_{ \pm}$, and expand the above Lie bracket as follows, where the indices $\alpha, \beta \in\{1,2\}$ and $i, j \in\{3, \ldots, 8\}$ :

$$
\begin{aligned}
{[\Psi(\varepsilon), \Psi(\varepsilon)]=} & \left(\bar{\varepsilon}_{+} \Gamma_{-} \varepsilon_{+}\right)\left(P_{+}-L_{12}\right)-\frac{1}{4}\left(\bar{\varepsilon}_{+} \Gamma^{i j} \boldsymbol{I} \Gamma_{-} \varepsilon_{+}\right) M_{i j} \\
& +\left(\bar{\varepsilon}_{-} \Gamma_{+} \varepsilon_{-}\right)\left(P_{-}+2 L_{12}\right)+\frac{1}{2}\left(\bar{\varepsilon}_{-} \Gamma^{i j} \boldsymbol{I} \Gamma_{+} \varepsilon_{-}\right) M_{i j} \\
& +2\left(\bar{\varepsilon}_{+} \Gamma^{\alpha} \varepsilon_{-}\right) P_{\alpha}+2\left(\bar{\varepsilon}_{+} \Gamma^{i} \varepsilon_{-}\right) P_{i}+4\left(\bar{\varepsilon}_{+} \Gamma^{\alpha} \boldsymbol{I} \varepsilon_{-}\right) L_{0 \alpha}-2\left(\bar{\varepsilon}_{+} \Gamma^{i} \boldsymbol{I} \varepsilon_{-}\right) M_{9 i}
\end{aligned}
$$

where we have defined $P_{+}=\frac{1}{2}\left(P_{9}+P_{0}\right)$ and $P_{-}=P_{9}-P_{0}$.

Let us now define a real $\mathbb{Z}_{2}$-graded vector space $E=E_{\overline{0}} \oplus E_{\overline{1}}$, where $E_{\overline{0}}$ is spanned by the symbols $\left(\xi^{\prime}, \zeta^{\prime}, J^{\prime}, M_{i j}^{\prime}, p_{\alpha}^{\prime}, q_{\alpha}^{\prime}, p_{i}^{\prime}, q_{i}^{\prime}\right)$ for $\alpha, \beta=1,2$ and $i, j=3, \ldots, 8$ and $E_{\overline{1}}$ is the isomorphic image of $\Psi^{\prime}: \Delta_{+}^{(9,1)} \rightarrow E_{\overline{1}}$. We will define a family $\Upsilon_{t}: E \rightarrow \mathfrak{s}$ of even $\mathbb{Z}_{2}$-graded linear maps by extending the following maps linearly:

$$
\begin{aligned}
\Upsilon_{t}\left(\xi^{\prime}\right) & =\frac{\mu}{12} t^{2}\left(P_{9}+P_{0}\right) & \Upsilon_{t}\left(p_{\alpha}^{\prime}\right) & =\frac{\mu}{6} t P_{\alpha} \\
\Upsilon_{t}\left(\zeta^{\prime}\right) & =\frac{\mu}{6}\left(P_{9}-P_{0}\right) & \Upsilon_{t}\left(p_{i}^{\prime}\right) & =\frac{\mu}{6} t P_{i} \\
\Upsilon_{t}\left(J^{\prime}\right) & =L_{12} & \Upsilon_{t}\left(q_{\alpha}^{\prime}\right) & =t L_{0 \alpha} \\
\Upsilon_{t}\left(M_{i j}^{\prime}\right) & =M_{i j} & \Upsilon_{t}\left(q_{i}^{\prime}\right) & =t M_{9 i}
\end{aligned}
$$


and where, for $\varepsilon \in \Delta_{+}^{(9,1)}$,

$$
\Upsilon_{t}\left(\Psi^{\prime}(\varepsilon)\right)= \begin{cases}\lambda t \Psi(\varepsilon), & \text { if } \varepsilon \in \operatorname{ker} \Gamma_{+} \\ \lambda \Psi(\varepsilon), & \text { if } \varepsilon \in \operatorname{ker} \Gamma_{-},\end{cases}
$$

with $\lambda^{2}=\frac{\mu}{6}$. (We tacitly assume $\mu>0$, but in fact the factor $\mu$ is inessential and can always be taken to be 1 , if nonzero.) It is clear by inspection that $\Upsilon_{t}$ defines a vector space isomorphism for any $t \neq 0$. For definiteness, let us take $t>0$. We may define a family of Lie brackets $[-,-]_{t}$ on $E$ by transporting the Lie bracket on $\mathfrak{s}$ via $\Upsilon_{t}$ :

$$
[x, y]_{t}:=\Upsilon_{t}^{-1}\left[\Upsilon_{t}(x), \Upsilon_{t}(y)\right]
$$

for $x, y \in E$. By construction, for every $t>0,\left(E,[-,-]_{t}\right)$ and $(\mathfrak{s},[-,-])$ are isomorphic Lie superalgebras. If the limit $t \rightarrow 0$ exists, then $\left(E,[-,-]_{0}\right)$ defines a Lie superalgebra, which is then a contraction of $(\mathfrak{s},[-,-])$. One checks that for the map $\Upsilon_{t}$ defined in equations (2.42) and (2.43), the limit $t \rightarrow 0$ of the $[-,-]_{t}$ bracket does exist and that the resulting bracket is precisely the one defined by equations $(2.29),(2.30),(2.34)$ (without the $\theta$ bracket) and (2.35), once we remove the primes from the symbols, and identify $\eta_{+}=\varepsilon_{+}$ and $\eta_{-}=\Gamma_{+} \varepsilon_{-}$.

Let us illustrate this with some examples. Firstly, let us consider the bracket $\left[q_{i}^{\prime}, p_{j}^{\prime}\right]$, which is given by

$$
\begin{aligned}
{\left[q_{i}^{\prime}, p_{j}^{\prime}\right] } & =\lim _{t \rightarrow 0} \Upsilon_{t}^{-1}\left[\Upsilon_{t}\left(q_{i}^{\prime}\right), \Upsilon_{t}\left(p_{j}^{\prime}\right)\right] \\
& =\lim _{t \rightarrow 0} \Upsilon_{t}^{-1}\left[t M_{9 i}, \frac{\mu}{6} t P_{j}\right] \\
& =\lim _{t \rightarrow 0} \frac{\mu}{6} t^{2} \Upsilon_{t}^{-1} \delta_{i j} P_{9} \\
& =\lim _{t \rightarrow 0} \delta_{i j} \frac{\mu}{6}\left(\frac{6}{\mu} \xi^{\prime}+\frac{3 t^{2}}{\mu} \zeta^{\prime}\right) \\
& =\delta_{i j} \xi^{\prime}
\end{aligned}
$$

which agrees with equation (2.29). Next we consider the bracket $\left[p_{\alpha}^{\prime}, \Psi^{\prime}\left(\varepsilon_{-}\right)\right]$, for $\varepsilon_{-} \in$ ker $\Gamma_{-}$, given by

$$
\begin{aligned}
{\left[p_{\alpha}^{\prime}, \Psi^{\prime}\left(\varepsilon_{-}\right)\right] } & =\lim _{t \rightarrow 0} \Upsilon_{t}^{-1}\left[\Upsilon_{t}\left(p_{\alpha}^{\prime}\right), \Upsilon_{t}\left(\Psi^{\prime}\left(\varepsilon_{-}\right)\right)\right] \\
& =\lim _{t \rightarrow 0} \Upsilon_{t}^{-1}\left[\frac{\mu}{6} t P_{\alpha}, \lambda \Psi\left(\varepsilon_{-}\right)\right] \\
& =\lim _{t \rightarrow 0} \frac{\mu \lambda}{6} \Upsilon_{t}^{-1} t \Psi\left(\Gamma_{\alpha} \nu \varepsilon_{-}\right) \\
& =\frac{\mu}{6} \Psi^{\prime}\left(\Gamma_{\alpha} I \Gamma_{+} \varepsilon_{-}\right)
\end{aligned}
$$

which shows that $\eta_{-}=\Gamma_{+} \varepsilon_{-}$for agreement with equation (2.34). Next, we consider the 
bracket $\left[\Psi^{\prime}\left(\varepsilon_{+}\right), \Psi^{\prime}\left(\varepsilon_{+}\right)\right]$, where $\varepsilon_{+} \in \operatorname{ker} \Gamma_{+}$, whose contraction is

$$
\begin{aligned}
{\left[\Psi^{\prime}\left(\varepsilon_{+}\right), \Psi^{\prime}\left(\varepsilon_{+}\right)\right] } & =\lim _{t \rightarrow 0} \Upsilon_{t}^{-1}\left[\Upsilon_{t}\left(\Psi^{\prime}\left(\varepsilon_{+}\right)\right), \Upsilon_{t}\left(\Psi^{\prime}\left(\varepsilon_{+}\right)\right)\right] \\
& =\lambda^{2} \lim _{t \rightarrow 0} t^{2} \Upsilon_{t}^{-1}\left[\Psi\left(\varepsilon_{+}\right), \Psi\left(\varepsilon_{+}\right)\right] \\
& =\frac{\mu}{6} \lim _{t \rightarrow 0} t^{2} \Upsilon_{t}^{-1}\left(\left(\bar{\varepsilon}_{+} \Gamma_{-} \varepsilon_{+}\right)\left(P_{+}-L_{12}\right)-\frac{1}{2}\left(\bar{\varepsilon}_{+} \Gamma^{i j} \boldsymbol{I} \Gamma_{-} \varepsilon_{+}\right) M_{i j}\right) \\
& =\frac{\mu}{6} \lim _{t \rightarrow 0}\left(\left(\bar{\varepsilon}_{+} \Gamma_{-} \varepsilon_{+}\right)\left(\frac{6}{\mu} \xi^{\prime}-t^{2} J^{\prime}\right)-\frac{1}{2}\left(\bar{\varepsilon}_{+} \Gamma^{i j} \boldsymbol{I} \Gamma_{-} \varepsilon_{+}\right) t^{2} M_{i j}^{\prime}\right) \\
& =\left(\bar{\varepsilon}_{+} \Gamma_{-} \varepsilon_{+}\right) \xi^{\prime},
\end{aligned}
$$

and $\left[\Psi^{\prime}\left(\varepsilon_{-}\right), \Psi^{\prime}\left(\varepsilon_{-}\right)\right]$, given by

$$
\begin{aligned}
{\left[\Psi^{\prime}\left(\varepsilon_{-}\right), \Psi^{\prime}\left(\varepsilon_{-}\right)\right] } & =\lim _{t \rightarrow 0} \Upsilon_{t}^{-1}\left[\Upsilon_{t}\left(\Psi^{\prime}\left(\varepsilon_{-}\right)\right), \Upsilon_{t}\left(\Psi^{\prime}\left(\varepsilon_{-}\right)\right)\right] \\
& =\lambda^{2} \lim _{t \rightarrow 0} \Upsilon_{t}^{-1}\left[\Psi\left(\varepsilon_{-}\right), \Psi\left(\varepsilon_{-}\right)\right] \\
& =\frac{\mu}{6} \lim _{t \rightarrow 0} \Upsilon_{t}^{-1}\left(\left(\bar{\varepsilon}_{-} \Gamma_{+} \varepsilon_{-}\right)\left(P_{-}+2 L_{12}\right)+\frac{1}{2}\left(\bar{\varepsilon}_{-} \Gamma^{i j} \boldsymbol{I} \Gamma_{+} \varepsilon_{-}\right) M_{i j}\right) \\
& =\frac{\mu}{6} \lim _{t \rightarrow 0}\left(\left(\bar{\varepsilon}_{-} \Gamma_{+} \varepsilon_{-}\right)\left(\frac{6}{\mu} \zeta^{\prime}+2 J^{\prime}\right)+\frac{1}{2}\left(\bar{\varepsilon}_{-} \Gamma^{i j} \boldsymbol{I} \Gamma_{+} \varepsilon_{-}\right) M_{i j}^{\prime}\right) \\
& =\left(\bar{\varepsilon}_{-} \Gamma_{+} \varepsilon_{-}\right)\left(\zeta^{\prime}+\frac{\mu}{3} J^{\prime}\right)+\frac{\mu}{12}\left(\bar{\varepsilon}_{-} \Gamma^{i j} \boldsymbol{I} \Gamma_{+} \varepsilon_{-}\right) M_{i j}^{\prime}
\end{aligned}
$$

which agree with the first line of equation (2.35), again using $\eta_{-}=\Gamma_{+} \varepsilon_{-}$.

Finally, we should remark that the infinitesimal homothety $\theta$ of the Cahen-Wallach background is not inherited from the Freund-Rubin backgrounds via the plane-wave limit, hence we are not obtaining the full conformal symmetry superalgebra as a contraction. Of course, this is not unexpected.

\subsubsection{Maximal superalgebras}

In [110] the notion of the maximal superalgebra of a supergravity background was introduced, generalising to non-flat backgrounds the M-algebra of [111]. Given a supergravity background with Killing superalgebra $\mathfrak{k}=\mathfrak{k}_{\overline{0}} \oplus \mathfrak{k}_{\overline{1}}$, the maximal superalgebra (should it exist) is defined to be Lie superalgebra $\mathfrak{m}=\mathfrak{m}_{\overline{0}} \oplus \mathfrak{m}_{\overline{1}}$, satisfying the following properties

1. $\mathfrak{m}_{\overline{1}}=\mathfrak{k}_{\overline{1}}$ and $\mathfrak{k}_{\overline{0}}$ is a Lie subalgebra of $\mathfrak{m}_{\overline{0}}$;

2. the odd-odd bracket is an isomorphism $\odot^{2} \mathfrak{m}_{\overline{1}} \cong \mathfrak{m}_{\overline{0}}$; and

3. the projection $\odot^{2} \mathfrak{m}_{\overline{1}} \rightarrow \mathfrak{k}_{\overline{0}}$ coincides with the odd-odd bracket of $\mathfrak{k}$ and the restriction to $\mathfrak{k}_{\overline{0}}$ of the bracket $\mathfrak{m}_{\overline{0}} \otimes \mathfrak{m}_{\overline{1}} \rightarrow \mathfrak{m}_{\overline{1}}$ is the $\mathfrak{k}_{\text {-bracket. }}$

In other words, writing $\mathfrak{m}_{\overline{0}}=\mathfrak{k}_{\overline{0}} \oplus \mathfrak{z} \overline{0}$, then the $\mathfrak{m}$-brackets $\left[\mathfrak{k}_{\overline{0}}, \mathfrak{k}_{\overline{0}}\right],\left[\mathfrak{k}_{\overline{0}}, \mathfrak{m}_{\overline{1}}\right]$ and the $\mathfrak{k}_{\overline{0}}-$ component of $\left[\mathfrak{m}_{\overline{1}}, \mathfrak{m}_{\overline{1}}\right]$ are, respectively, the $\left[\mathfrak{k}_{\overline{0}}, \mathfrak{k}_{\overline{0}}\right],\left[\mathfrak{k}_{\overline{0}}, \mathfrak{k}_{\overline{1}}\right]$ and $\left[\mathfrak{k}_{\overline{1}}, \mathfrak{k}_{\overline{1}}\right]$ brackets of $\mathfrak{k}$, and only the brackets involving $\mathfrak{z}_{\overline{0}}$ are genuinely new.

As reviewed in [110], it follows from ([112], appendix A) that any Lie superalgebra satisfying (2) - in particular, the maximal superalgebra of a background - is uniquely 
determined by some $\omega \in\left(\wedge^{2} \mathfrak{m}_{1}^{*}\right)^{\mathfrak{m}_{0}} \subset\left(\wedge^{2} \mathfrak{k}_{1}^{*}\right)^{\mathfrak{k}_{0}}$. Indeed, by (2) above, if $Q_{a}$ is a basis for $\mathfrak{m}_{\overline{1}}, Z_{a b}:=\left[Q_{a}, Q_{b}\right]$ is a basis for $\mathfrak{m}_{\overline{0}}$ and the Lie brackets in this basis are given by

$$
\begin{aligned}
{\left[Z_{a b}, Q_{c}\right] } & =\omega_{a c} Q_{b}+\omega_{b c} Q_{a} \\
{\left[Z_{a b}, Z_{c d}\right] } & =\omega_{a c} Z_{b d}+\omega_{b c} Z_{a d}+\omega_{a d} Z_{b c}+\omega_{b d} Z_{a c}
\end{aligned}
$$

where $\omega_{a b}:=\omega\left(Q_{a}, Q_{b}\right)$ and where the second equation follows from the first, using the Jacobi identity and the definition of $Z_{a b}$. In this section we explore the maximal superalgebras of the maximally supersymmetric backgrounds.

First of all, we show that the Cahen-Wallach background does not admit a maximal superalgebra. The proof is virtually identical to the one for the Cahen-Wallach vacua of eleven-dimensional and type IIB Poincaré supergravities in [110]. As explained in ([110], section 3), for any maximal superalgebra $\mathfrak{m}, \mathfrak{k}_{\overline{0}}$ acts trivially on the radical $\mathfrak{k}_{\overline{1}}^{\perp}$ of the skewsymmetric bilinear form $\omega$ characterising $\mathfrak{m}$. Now, inspecting equation (2.34) we see that $\zeta=\partial_{-}$acts semisimply on $\mathfrak{k}_{\overline{1}}$ with nonzero eigenvalues, so that $\mathfrak{k}_{\overline{1}}^{\mathfrak{k}_{\overline{0}}}=0$. Therefore $\omega$, having trivial radical, must be symplectic and hence, from equation (2.49), it follows that $\mathfrak{m}$ must have trivial centre. But now notice that $\xi=\partial_{+}$acts trivially on $\mathfrak{m}_{\overline{1}}$ and hence it is central in $\mathfrak{m}$, thus contradicting the existence of a maximal superalgebra for the Cahen-Wallach background.

Next we discuss the two maximally supersymmetric Freund-Rubin backgrounds $A d S_{7} \times S^{3}$ and $A d S_{3} \times S^{7}$. Here it is convenient to again think of their Killing superalgebras as different real forms of the same complex Lie superalgebra. At the same time we must make a distinction between the symmetry superalgebra $\mathfrak{s}^{\mathbb{C}}$ and the Killing superalgebra, which is the ideal $\mathfrak{k}^{\mathbb{C}}$ of $\mathfrak{s}^{\mathbb{C}}$ generated by $\mathfrak{k}_{1}^{\mathbb{C}}$. For the Freund-Rubin backgrounds, $\mathfrak{s}^{\mathbb{C}}$ is strictly larger, containing a simple ideal isomorphic to $\mathfrak{s l}_{2}(\mathbb{C})$, which acts trivially on the Killing spinors.

In this case, we have $\mathfrak{k}_{\overline{0}}^{\mathbb{C}} \cong \mathfrak{s l}_{2}(\mathbb{C}) \oplus \mathfrak{s o}_{8}(\mathbb{C})$ and $\mathfrak{k}_{\overline{1}}^{\mathbb{C}} \cong \Delta^{\mathbb{C}} \otimes \Delta_{+}^{(8, \mathbb{C})}$ as an $\mathfrak{k}_{\overline{0}}^{\mathbb{C}}$-module, where $\Delta^{\mathbb{C}}$ is the defining representation of $\mathfrak{s l}_{2}(\mathbb{C})$ and the tensor product is over $\mathbb{C}$. There is precisely one invariant skew-symmetric bilinear form (up to scale) on $\mathfrak{k}_{\overline{1}}^{\mathbb{C}}$ : it is the product of the $\mathfrak{s l}_{2}(\mathbb{C})$-invariant complex symplectic structure $\langle-,-\rangle$ on $\Delta^{\mathbb{C}}$ and the $\mathfrak{s o}_{8}(\mathbb{C})$-invariant complex orthogonal structure $(-,-)$ on $\Delta_{+}^{(8, \mathbb{C})}$. It is clearly nondegenerate, hence complex symplectic. Therefore if $\mathfrak{k}^{\mathbb{C}}$ admits a maximal superalgebra, it has to be the complexification $\mathfrak{o s p}(1 \mid 16)^{\mathbb{C}}$ of $\mathfrak{o s p}(1 \mid 16)$. On the other hand, it is shown in [106] that $\mathfrak{o s p}(1 \mid 16)$ is the unique real form of $\mathfrak{o s p}(1 \mid 16)^{\mathbb{C}}$, so if 'maximisation' were to commute with complexification, we would conclude that the maximal superalgebras of the Freund-Rubin backgrounds would be isomorphic to $\mathfrak{o s p}(1 \mid 16)$. We do not have such a result at our disposal and it is unlikely that such a general result actually exists since not every Lie superalgebra can be 'maximised', as illustrated by the Killing superalgebra of the Cahen-Wallach backgrounds. This means we need to work harder.

We start by showing that the maximal superalgebra of $\mathfrak{k}^{\mathbb{C}}$ is indeed isomorphic to $\mathfrak{o s p}(1 \mid 16)^{\mathbb{C}}$, following the construction in [110], mutatis mutandis. First of all, let us de- 
compose the symmetric square of $\mathfrak{m}_{\overline{1}}^{\mathbb{C}}$ into irreducible representations of $\mathfrak{k} \frac{\mathbb{C}}{0}$ :

$$
\begin{aligned}
\odot^{2} \mathfrak{m}_{\overline{1}}^{\mathbb{C}} & =\odot^{2}\left(\Delta^{\mathbb{C}} \otimes \Delta_{+}^{(8, \mathbb{C})}\right) \\
& =\left(\odot^{2} \Delta^{\mathbb{C}} \otimes \odot^{2} \Delta_{+}^{(8, \mathbb{C})}\right) \oplus\left(\wedge^{2} \Delta^{\mathbb{C}} \otimes \wedge^{2} \Delta_{+}^{(8, \mathbb{C})}\right) \\
& =\left(\odot^{2} \Delta^{\mathbb{C}} \otimes\left(\mathbb{C} \oplus \odot_{0}^{2} \Delta_{+}^{(8, \mathbb{C})}\right)\right) \oplus\left(\mathbb{C} \otimes \wedge^{2} \Delta_{+}^{(8, \mathbb{C})}\right) \\
& =\odot^{2} \Delta^{\mathbb{C}} \oplus \wedge^{2} \Delta_{+}^{(8, \mathbb{C})} \oplus\left(\odot^{2} \Delta^{\mathbb{C}} \otimes \odot_{0}^{2} \Delta_{+}^{(8, \mathbb{C})}\right) \\
& \cong \mathfrak{s l}_{2}(\mathbb{C}) \oplus \mathfrak{s o}_{8}(\mathbb{C}) \oplus \mathfrak{z} \overline{0}
\end{aligned}
$$

which defines $\mathfrak{z}_{\overline{0}}^{\mathbb{C}}$. Except for the $\mathfrak{z}_{0}^{\mathbb{C}}$, this is precisely the odd-odd bracket of $\mathfrak{k}^{\mathbb{C}}$. It follows from the discussion in ([110], section 4.3) that the action of $\mathfrak{k}_{\overline{0}}$ on $\mathfrak{k}_{\overline{1}}$, when viewed through the lens of the cone construction, is via the Clifford action of the parallel 2-forms on the cones of $A d S_{p}$ and $S^{q}$ to which the special Killing 1-forms in $\mathfrak{k}_{\overline{0}}$ lift. Therefore we extend this Clifford action to all of $\mathfrak{m}_{\overline{0}}$, which also lift as parallel forms to the cones. This complexifies and gives the following construction of $\mathfrak{m}^{\mathbb{C}}$; although we prefer to use a different basis, which unfortunately obscures the embedding of $\mathfrak{k}_{\overline{0}}^{\mathbb{C}}$ into $\mathfrak{m}_{\overline{0}}^{\mathbb{C}}$.

If $\psi_{1} \otimes \varphi_{1}, \psi_{2} \otimes \varphi_{2} \in \mathfrak{m}_{1}^{\mathbb{C}}$, their symplectic inner product is given by

$$
\omega\left(\psi_{1} \otimes \varphi_{1}, \psi_{2} \otimes \varphi_{2}\right)=\left\langle\psi_{1}, \psi_{2}\right\rangle\left(\varphi_{1}, \varphi_{2}\right) .
$$

We define the following rank-1 endomorphisms of $\Delta^{\mathbb{C}}$ and $\Delta_{+}^{(8, \mathbb{C})}$ :

$$
\psi_{1} \bar{\psi}_{2}:=\left\langle\psi_{2},-\right\rangle \psi_{1} \quad \text { and } \quad \varphi_{1} \bar{\varphi}_{2}:=\left(\varphi_{2},-\right) \varphi_{1}
$$

and we define the odd-odd bracket in $\mathfrak{m}^{\mathbb{C}}$ via

$$
\left[\psi_{1} \otimes \varphi_{1}, \psi_{2} \otimes \varphi_{2}\right]=\psi_{1} \bar{\psi}_{2} \otimes \varphi_{1} \bar{\varphi}_{2}+\psi_{2} \bar{\psi}_{1} \otimes \varphi_{2} \bar{\varphi}_{1}
$$

By a judicious use of the Fierz identities, the rank-1 endomorphisms above can be expressed in terms of the standard basis for the Clifford algebra in terms of exterior forms, and in this way clarify the embedding $\mathfrak{k}_{\overline{0}}^{\mathbb{C}} \subset \mathfrak{m}_{\overline{0}}^{\mathbb{C}}$, but we have no need to do that. The action of $\mathfrak{m}_{\overline{0}}^{\mathbb{C}}$ on $\mathfrak{m}_{\overline{1}}^{\mathbb{C}}$ is given simply by the Clifford action, which is

$$
\begin{aligned}
{\left[\psi_{1} \bar{\psi}_{2} \otimes \varphi_{1} \bar{\varphi}_{2}+\psi_{2} \bar{\psi}_{1} \otimes \varphi_{2} \bar{\varphi}_{1}, \psi_{3} \otimes \varphi_{3}\right]=} & \left\langle\psi_{2}, \psi_{3}\right\rangle\left(\varphi_{2}, \varphi_{3}\right) \psi_{1} \otimes \varphi_{1}+\left\langle\psi_{1}, \psi_{3}\right\rangle\left(\varphi_{1}, \varphi_{3}\right) \psi_{2} \otimes \varphi_{2} \\
= & \omega\left(\psi_{2} \otimes \varphi_{2}, \psi_{3} \otimes \varphi_{3}\right) \psi_{1} \otimes \varphi_{1} \\
& +\omega\left(\psi_{1} \otimes \varphi_{1}, \psi_{3} \otimes \varphi_{3}\right) \psi_{2} \otimes \varphi_{2}
\end{aligned}
$$

which agrees with the first equation in (2.49), showing that indeed $\mathfrak{m}^{\mathbb{C}} \cong \mathfrak{o s p}(1 \mid 16)^{\mathbb{C}}$.

How about the maximal subalgebras of $\mathfrak{k}\left(A d S_{3} \times S^{7}\right)$ and $\mathfrak{k}\left(S^{3} \times A d S_{7}\right)$ ? Let $\mathfrak{k}$ be one of these Killing superalgebras. It is a real form of $\mathfrak{k}^{\mathbb{C}}$, so in particular $\mathfrak{k}_{\overline{1}}$ is the real subspace of $\mathfrak{k}_{\overline{1}}^{\mathbb{C}}$ defined by a $\mathfrak{k}_{\overline{0}}$-invariant conjugation. Now consider $\mathfrak{k}_{\overline{1}}$ as a real subspace of $\mathfrak{m}_{\overline{1}}^{\mathbb{C}}$. It generates a real subalgebra of $\mathfrak{m}^{\mathbb{C}}$, which satisfies property (2) of a maximal subalgebra because the restriction to $\mathfrak{k}_{\overline{1}}$ of the odd-odd bracket is an isomorphism onto its image. This means that the brackets are of the form (2.49) with $\omega$ being the restriction 
of the complex-symplectic form on $\mathfrak{m}_{\overline{1}}^{\mathbb{C}}$ to the real subspace $\mathfrak{k}_{\overline{1}}$. The other properties for a maximal subalgebra are satisfied because $\mathfrak{k}^{\mathbb{C}}$ is the complexification of $\mathfrak{k}$. Therefore we see that $\mathfrak{k}$ admits a maximal superalgebra, but to identify it we need to understand the restriction of $\omega$ to $\mathfrak{k}_{\overline{1}}$. It pays to be a little bit more general.

Let $(E, \omega)$ be a complex symplectic vector space. Let $\mathfrak{g}$ be a Lie algebra, whose complexification $\mathfrak{g}^{\mathbb{C}}$ acts on $E$ preserving $\omega$. Now suppose that $c$ is a $\mathfrak{g}$-invariant conjugation on $E$ and let $E^{\mathbb{R}}$ be its fixed (real) subspace; that is, $E=E^{\mathbb{R}} \otimes_{\mathbb{R}} \mathbb{C}$. Because $c$ is $\mathfrak{g}$-invariant, $\mathfrak{g}$ acts on $E^{\mathbb{R}}$. Now, $\omega$ restricts to a real skewsymmetric bilinear form $\omega^{\mathbb{R}}$ on $E^{\mathbb{R}}$. Since $\omega$ is

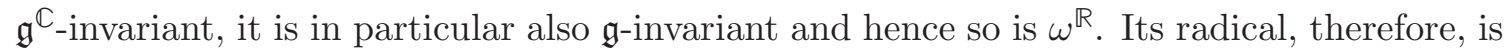
a $\mathfrak{g}$-submodule of $E^{\mathbb{R}}$. Now suppose that $E^{\mathbb{R}}$ is irreducible as a $\mathfrak{g}$-module. Then the radical of $\omega^{\mathbb{R}}$ must either be trivial, in which case $\omega^{\mathbb{R}}$ is a symplectic form, or it must be all of $E^{\mathbb{R}}$, in which case $\omega^{\mathbb{R}}=0$.

Now let us apply this to our situation, with the role of $(E, \omega)$ played by $\left(\mathfrak{k}_{\overline{1}}^{\mathbb{C}}, \omega\right)$. We have that $\mathfrak{k}_{\overline{1}}$ is an irreducible module of $\mathfrak{k}_{\overline{0}}$, so that the restriction of $\omega$ to $\mathfrak{k}_{\overline{1}}$ is either symplectic or zero. But it cannot be zero, because otherwise $\mathfrak{k}_{\overline{0}}$ would be central and in particular, an abelian Lie algebra. Therefore we conclude that $\omega$ restricts to a symplectic form on $\mathfrak{k}_{\overline{1}}$ and hence $\mathfrak{k}_{\overline{1}}$ generates a maximal superalgebra isomorphic to $\mathfrak{o s p}(1 \mid 16)$.

\subsection{F1-string and NS5-brane, Weyl transformations and near-horizon limits}

Backgrounds which solve (2.3) for precisely eight linearly independent supersymmetry parameters $\epsilon$ are called half-BPS. Two well-known half-BPS backgrounds in ten dimensions are the F1-string [113] and the NS5-brane [114]. They solve (2.3) with $\mathrm{d} H=0$ and $G \epsilon=\frac{1}{2} H \epsilon$ and thus define half-BPS backgrounds of type I supergravity in ten dimensions. To define them, it is convenient to write $g_{\mathbb{R}^{p, q}}$ and $\operatorname{vol}_{\mathbb{R}^{p, q}}$ for the canonical flat metric and volume form on $\mathbb{R}^{p, q}$.

The F1-string background has metric and three-form given by

$$
g=\mathrm{e}^{2 \Phi} g_{\mathbb{R}^{1,1}}+g_{\mathbb{R}^{8}}, \quad H=\operatorname{vol}_{\mathbb{R}^{1,1}} \wedge \mathrm{de}^{2 \Phi},
$$

where $\mathrm{e}^{-2 \Phi}$ is a harmonic function on $\mathbb{R}^{8}$ so that $\mathrm{d}\left(\mathrm{e}^{-2 \Phi} \star H\right)=0$. For example, thinking of $\mathbb{R}^{8}$ as a cone over $S^{7}$ with radial coordinate $r$, one can take $\mathrm{e}^{-2 \Phi}=1+\frac{\left|k_{2}\right|}{r^{6}}$ for some constant $k_{2}$. The supersymmetry parameter is given by

$$
\epsilon=\mathrm{e}^{\Phi / 2} \epsilon_{0}, \quad \operatorname{vol}_{\mathbb{R}^{1,1}} \epsilon_{0}=\epsilon_{0},
$$

where $\epsilon_{0}$ is a constant positive chirality Majorana-Weyl spinor on $\mathbb{R}^{9,1}$.

Now consider the Weyl transformation (with $\Omega=\mathrm{e}^{-\Phi / 3}$ ) of the F1-string that defines a solution of (2.4). This is a new half-BPS background of conformal (but not Poincaré) supergravity in ten dimensions. Its 'near-horizon' limit is defined by taking the radial coordinate $r \rightarrow 0$, which recovers precisely the maximally supersymmetric $A d S_{3} \times S^{7}$ background obtained in section 2.4 (identifying $\left|k_{2}\right|^{-1 / 3}=R / 18$ ).

The NS5-brane background has metric and three-form given by

$$
g=g_{\mathbb{R}^{5,1}}+\mathrm{e}^{2 \Phi} g_{\mathbb{R}^{4}}, \quad H=-\star_{\mathbb{R}^{4}} \mathrm{de}^{2 \Phi},
$$


where $\mathrm{e}^{2 \Phi}$ is a harmonic function on $\mathbb{R}^{4}$ so that $\mathrm{d} H=0$. For example, thinking of $\mathbb{R}^{4}$ as a cone over $S^{3}$ with radial coordinate $r$, one can take $\mathrm{e}^{2 \Phi}=1+\frac{\left|k_{6}\right|}{r^{2}}$ for some constant $k_{6}$. The supersymmetry parameter is given by

$$
\epsilon=\epsilon_{0}, \quad \operatorname{vol}_{\mathbb{R}^{4}} \epsilon_{0}=\epsilon_{0},
$$

where $\epsilon_{0}$ is a constant positive chirality Majorana-Weyl spinor on $\mathbb{R}^{9,1}$.

The near-horizon limit of the NS5-brane defines a metric on $\mathbb{R}^{5,1} \times \mathbb{R}_{+} \times S^{3}$ and is therefore not conformally equivalent to the maximally supersymmetric $A d S_{7} \times S^{3}$ background obtained section 2.4. However, it is important to stress that any choice of function $\mathrm{e}^{2 \Phi}$ on $\mathbb{R}^{4}$ for the NS5-brane defines a half-BPS background of conformal supergravity in ten dimensions. Let us therefore not assume that $\mathrm{e}^{2 \Phi}$ is harmonic on $\mathbb{R}^{4}$ and perform the Weyl transformation (with $\Omega=\mathrm{e}^{-\Phi / 3}$ ) to define a solution of (2.4). Now, for this new half-BPS background of conformal supergravity, taking $\mathrm{e}^{2 \Phi}=1+\frac{\left|k_{6}^{\prime}\right|}{r^{3}}$ for some constant $k_{6}^{\prime}$ (which is not harmonic on $\mathbb{R}^{4}$ ), one recovers in the near-horizon limit precisely the maximally supersymmetric $A d S_{7} \times S^{3}$ background obtained in section 2.4 (identifying $\left.\left|k_{6}^{\prime}\right|^{-2 / 3}=-2 R / 9\right)$.

\section{Yang-Mills supermultiplet}

The on-shell Yang-Mills supermultiplet in ten dimensions contains a bosonic gauge field $A_{\mu}$ and a fermionic Majorana-Weyl spinor $\lambda$ (we take $\lambda$ with positive chirality, i.e., $\Gamma \lambda=\lambda$ ). Both fields are valued in a real Lie algebra $\mathfrak{g}$ with invariant inner product $(-,-)$.

The supersymmetry variations are

$$
\begin{aligned}
\delta_{\epsilon} A_{\mu} & =\bar{\epsilon} \Gamma_{\mu} \lambda \\
\delta_{\epsilon} \lambda & =-F \epsilon,
\end{aligned}
$$

where $\epsilon$ is a bosonic Majorana-Weyl spinor with positive chirality. The variations in (3.1) are Weyl-invariant provided $\left(A_{\mu}, \lambda, \epsilon\right)$ are assigned weights $\left(0,-\frac{3}{2}, \frac{1}{2}\right)$. For a bosonic supersymmetric conformal supergravity background, the supersymmetry parameter $\epsilon$ obeys (2.3).

Up to boundary terms, the lagrangian

$$
L=\mathrm{e}^{-2 \Phi}\left(-\frac{1}{4}\left(F_{\mu \nu}, F^{\mu \nu}\right)-\frac{1}{2}(\bar{\lambda}, \not D \lambda)+\frac{1}{8}(\bar{\lambda}, H \lambda)+\frac{1}{2} H^{\mu \nu \rho}\left(A_{\mu}, \partial_{\nu} A_{\rho}+\frac{1}{3}\left[A_{\nu}, A_{\rho}\right]\right)\right),
$$

is invariant under (3.1), for any $\epsilon$ obeying (2.3). (This result was noted in [23] for the subclass of bosonic supersymmetric backgrounds of type I supergravity in ten dimensions.) The prefactor $\mathrm{e}^{-2 \Phi}$ acts as an effective gauge coupling in (3.2). For generic backgrounds with $H \neq 0$, notice that rigid supersymmetry necessitates both a mass term for $\lambda$ and a Chern-Simons coupling for the gauge field. Closure of $\mathrm{e}^{-2 \Phi} \star H$ ensures that the ChernSimons coupling is gauge-invariant.

Squaring $\delta_{\epsilon}$ in (3.1) with $\epsilon$ subject to (2.3) gives

$$
\begin{aligned}
\delta_{\epsilon}^{2} A_{\mu} & =-F_{\mu \nu} \xi^{\nu}=\mathscr{L}_{\xi} A_{\mu}+D_{\mu} \Lambda \\
\delta_{\epsilon}^{2} \lambda & =\mathscr{L}_{\xi} \lambda+\frac{1}{2} G_{\xi} \lambda+[\lambda, \Lambda]+\left(\epsilon \bar{\epsilon}-\frac{1}{2} \xi\right)\left(\not D \lambda-G \lambda-\frac{1}{4} H \lambda\right),
\end{aligned}
$$


where $\xi^{\mu}=\bar{\epsilon} \Gamma^{\mu} \epsilon$ and $\Lambda=-A_{\xi}$. The Lie derivative $\mathscr{L}_{X}$ along a conformal Killing vector $X$ is defined such that $\mathscr{L}_{X} A_{\mu}=X^{\nu} \partial_{\nu} A_{\mu}+\left(\partial_{\mu} X^{\nu}\right) A_{\nu}$ and $\mathscr{L}_{X} \lambda=\nabla_{X} \lambda+\frac{1}{4}\left(\nabla_{\mu} X_{\nu}\right) \Gamma^{\mu \nu} \lambda$. The final term on the right hand side of $\delta_{\epsilon}^{2} \lambda$ in (3.3) vanishes using the field equation $\not D \lambda-G \lambda-\frac{1}{4} H \lambda=0$ for $\lambda$, derived from (3.2). Thus, on-shell, it follows that

$$
\delta_{\epsilon}^{2}=\mathscr{L}_{\xi}+w \sigma_{\xi}+\delta_{\Lambda}
$$

on any field in the Yang-Mills supermultiplet with Weyl weight $w$, where $\sigma_{\xi}=-\frac{1}{10} \nabla_{\mu} \xi^{\mu}=$ $-\frac{1}{3} \partial_{\xi} \Phi$ is the parameter for a Weyl variation and $\delta_{\Lambda}$ denotes a gauge variation with parameter $\Lambda=-A_{\xi}$.

A novel (partially) off-shell formulation of supersymmetric Yang-Mills theory on $\mathbb{R}^{9,1}$ was obtained by Berkovits in [94] (see also [95, 96]). To match the 16 off-shell fermionic degrees of freedom of $\lambda$, the 9 off-shell degrees of freedom of $A_{\mu}$ are supplemented by 7 bosonic auxiliary scalar fields $Y_{i}$ (where $i=1, \ldots, 7$ ). All fields are $\mathfrak{g}$-valued. The supersymmetry parameter $\epsilon$ is also supplemented by seven linearly independent bosonic Majorana-Weyl spinors $\theta_{i}$, each with the same positive chirality as $\epsilon$. The index $i$ corresponds to the vector representation of the $\mathfrak{s p i n}(7)$ factor in the isotropy algebra $\mathfrak{s p i n}(7) \ltimes \mathbb{R}^{8}$ of $\epsilon$.

Now consider the following supersymmetry variations for the partially off-shell YangMills supermultiplet on a bosonic supersymmetric conformal supergravity background

$$
\begin{aligned}
\delta_{\epsilon} A_{\mu} & =\bar{\epsilon} \Gamma_{\mu} \lambda \\
\delta_{\epsilon} \lambda & =-F \epsilon+Y_{i} \theta_{i} \\
\delta_{\epsilon} Y_{i} & =\bar{\theta}_{i}\left(\not D \lambda-G \lambda-\frac{1}{4} H \lambda\right) .
\end{aligned}
$$

Under the Weyl transformation $g_{\mu \nu} \mapsto \Omega^{2} g_{\mu \nu}, H_{\mu \nu \rho} \mapsto \Omega^{2} H_{\mu \nu \rho}, \Phi \mapsto \Phi+3 \ln \Omega$ of the background data that was described in section 2.1, the supersymmetry variations in (3.5) are invariant provided we assign $\left(A_{\mu}, \lambda, Y_{i}\right)$ their canonical weights $\left(0,-\frac{3}{2},-2\right)$, with $\epsilon$ and $\theta_{i}$ both having weight $\frac{1}{2}$. (The Weyl transformation $\not \mapsto \mapsto \Omega^{-11 / 2} \not \Omega^{9 / 2}$ of the Dirac operator in ten dimensions can be used to prove this for $\delta_{\epsilon} Y_{i}$.)

The supersymmetry parameters $\epsilon$ and $\theta_{i}$ are related such that

$$
\bar{\epsilon} \Gamma_{\mu} \theta_{i}=0, \quad \bar{\theta}_{i} \Gamma_{\mu} \theta_{j}=\delta_{i j} \xi_{\mu} \quad \text { and } \quad \epsilon \bar{\epsilon}+\theta_{i} \bar{\theta}_{i}=\frac{1}{2} \xi
$$

Squaring (3.5) subject to (3.6) gives precisely (3.4) on $A_{\mu}$ and $\lambda$, without needing to impose the field equation for $\lambda$. Moreover,

$$
\delta_{\epsilon}^{2} Y_{i}=\mathscr{L}_{\xi} Y_{i}-2 \sigma_{\xi} Y_{i}+\left[Y_{i}, \Lambda\right]+\Upsilon_{i j} Y_{j},
$$

where $\Upsilon_{i j}=\bar{\theta}_{[i} \not \nabla \theta_{j]}-\frac{1}{4} \bar{\theta}_{i} H \theta_{j}$ corresponds to a $\mathfrak{s p i n}(7)$ rotation.

Up to boundary terms, the lagrangian

$$
\begin{aligned}
L= & \mathrm{e}^{-2 \Phi}\left(-\frac{1}{4}\left(F_{\mu \nu}, F^{\mu \nu}\right)-\frac{1}{2}(\bar{\lambda}, \not D \lambda)+\frac{1}{2}\left(Y_{i}, Y_{i}\right)+\frac{1}{8}(\bar{\lambda}, H \lambda)\right. \\
& \left.+\frac{1}{2} H^{\mu \nu \rho}\left(A_{\mu}, \partial_{\nu} A_{\rho}+\frac{1}{3}\left[A_{\nu}, A_{\rho}\right]\right)\right),
\end{aligned}
$$


is invariant under (3.5). It is also manifestly invariant under $\mathfrak{s p i n}(7)$ rotations of the auxiliary fields. Moreover, the integral of (3.8) is Weyl-invariant with respect to the aforementioned transformation rules for fields and background data.

Of all the bosonic supersymmetric conformal supergravity backgrounds the Yang-Mills supermultiplet above can be defined upon, the maximally supersymmetric $A d S_{3} \times S^{7}$ and $A d S_{7} \times S^{3}$ Freund-Rubin backgrounds classified in section 2.4 are perhaps the most compelling. In particular, it would interesting to explore whether the Yang-Mills supermultiplet on these conformal supergravity backgrounds admits a consistent truncation that would recover one of the theories described in [13, 22, 24, 76, 92]. The relevant theories in [13] (or [24]) would follow by dimensionally reducing the on-shell (or partially off-shell) Yang-Mills supermultiplet on $\mathbb{R}^{9,1}$ to some lower dimension $d$ equal to either 7 or 3 , before deforming the resulting supermultiplet in dimension $d$ in such a way that it retains rigid supersymmetry on a curved space admitting the maximum number of real or imaginary Killing spinors, i.e., either $A d S_{d}$ or $S^{d}$. The deformation involves introducing several nonminimal couplings that do not seem to figure in (3.1) and (3.2), though this discrepancy may be the result of a non-standard reduction along some subset of Killing vectors of $S^{10-d}$ or $A d S_{10-d}$ that is necessary for the conformal supergravity background instead of along the obvious translations in $\mathbb{R}^{10-d}$ or $\mathbb{R}^{9-d, 1}$, as in $[13,24]$. We leave this question for future work.

\section{Lifting to eleven dimensions}

It should not have gone unnoticed that the supersymmetric backgrounds of conformal supergravity in ten dimensions that we have been discussing bear a striking resemblance to supersymmetric backgrounds of Poincaré supergravity in eleven dimensions. For instance, each maximally supersymmetric background obtained in section 2.4 has an obvious maximally supersymmetric counterpart in Theorem 1 of [100]. Moreover, the structure of the half-BPS string and five-brane backgrounds obtained in section 2.5 is virtually identical to that of the well-known half-BPS M2-brane and M5-brane solutions of Poincaré supergravity in eleven dimensions.

This empirical evidence hints at an embedding of (at least some) supersymmetric backgrounds of ten-dimensional conformal supergravity in supersymmetric solutions of elevendimensional Poincaré supergravity. Of course, this would be distinct from the well-known Kaluza-Klein reduction along a spacelike Killing vector for supergravity backgrounds in eleven dimensions, yielding backgrounds of type IIA supergravity in ten dimensions. After a brief synopsis of the defining conditions for bosonic supersymmetric backgrounds and solutions of eleven-dimensional Poincaré supergravity, we shall spend the rest of this final section investigating a few different types of embedding for some of the backgrounds of ten-dimensional conformal supergravity that we have already encountered. This will begin with a review of the Kaluza-Klein embedding of supersymmetric backgrounds of type I supergravity. We will then describe a novel 'equatorial' embedding for the maximally supersymmetric Freund-Rubin backgrounds of ten-dimensional conformal supergravity in their eleven-dimensional counterparts. Finally, we will describe the embedding of the half- 
BPS string and five-brane backgrounds of ten-dimensional conformal supergravity and show how to recover the maximally supersymmetric Freund-Rubin backgrounds via delocalisation and near-horizon limits.

\subsection{Supersymmetric solutions in eleven dimensions}

The bosonic fields of Poincaré supergravity in eleven dimensions consist of a metric $\hat{g}$ and a closed four-form $\hat{F}$. Following the conventions of [100], a bosonic supersymmetric background is given by a solution of

$$
\hat{\nabla}_{M} \hat{\epsilon}=-\frac{1}{24} \hat{\Gamma}_{M} \hat{F} \hat{\epsilon}+\frac{1}{8} \hat{F} \hat{\Gamma}_{M} \hat{\epsilon}
$$

where $\hat{\epsilon}$ is a Majorana spinor in eleven dimensions. Any such background is called a supersymmetric solution if it also obeys the field equations

$$
\begin{aligned}
\hat{R}_{M N} & =\frac{1}{12} \hat{F}_{M A B C} \hat{F}_{N}^{A B C}-\frac{1}{144} \hat{g}_{M N} \hat{F}_{A B C D} \hat{F}^{A B C D} \\
\mathrm{~d} \hat{\star} \hat{F} & =-\frac{1}{2} \hat{F} \wedge \hat{F} .
\end{aligned}
$$

Note that both (4.1) and (4.2) are invariant under the homothety $(\hat{g}, \hat{F}) \mapsto\left(\alpha^{2} \hat{g}, \alpha^{3} \hat{F}\right)$, for any $\alpha \in \mathbb{R}^{\times}$.

\subsection{Kaluza-Klein embedding of supersymmetric type I backgrounds}

It is well-known that any supersymmetric solution of type IIA supergravity in ten dimensions can be uplifted to a supersymmetric solution of supergravity in eleven dimensions via the 'string-frame' Kaluza-Klein ansatz. This recovers only the subset of supersymmetric solutions of supergravity in eleven dimensions which admit a spacelike Killing vector $\xi$ with $\mathscr{L}_{\xi} \hat{F}=0$. At least locally, one can write $\xi=\partial_{z}$ in terms of the eleventh coordinate $z$.

Now consider the following special case of the aforementioned ansatz:

$$
\begin{aligned}
\hat{g} & =\mathrm{e}^{4 \Phi / 3}(\mathrm{~d} z)^{2}+\mathrm{e}^{-2 \Phi / 3} g \\
\hat{F} & =\mathrm{d} z \wedge H,
\end{aligned}
$$

in terms of a metric $g$, a function $\Phi$ and a three-form $H$ in ten dimensions. It follows that $\hat{\star} \hat{F}=\mathrm{e}^{-2 \Phi} \star H$. Plugging (4.3) into the second field equation in (4.2) therefore gives $\mathrm{d}\left(\mathrm{e}^{-2 \Phi} \star H\right)=0$. It also follows that $\mathrm{d} H=0$ since $\hat{F}$ is closed.

The ansatz (4.3) allows one to define an idempotent element $\mathbf{I}=\mathrm{e}^{-2 \Phi / 3} \hat{\Gamma}_{z}$ which anticommutes with every $\hat{\Gamma}_{\mu}$ (where $\mu$ is any index $M \neq z$ ). If $\hat{\epsilon}=\mathbf{I} \hat{\epsilon}$ then it can be identified with a positive chirality Majorana-Weyl spinor $\mathrm{e}^{-\Phi / 6} \epsilon$ in ten dimensions. Assuming this to be the case then plugging (4.3) into (4.1) gives

$$
\begin{aligned}
\nabla_{\mu} \epsilon & =\frac{1}{6} \Gamma_{\mu} G \epsilon+\frac{1}{24} \Gamma_{\mu} H \epsilon+\frac{1}{8} H \Gamma_{\mu} \epsilon \\
G \epsilon & =\frac{1}{2} H \epsilon,
\end{aligned}
$$

with $\partial_{z} \epsilon=0$ and $G=\mathrm{d} \Phi$. The first condition in (4.4) is identified with (2.3) provided $\mathrm{d}\left(\mathrm{e}^{-2 \Phi} \star H\right)=0$. Since $H$ is closed, the second condition in (4.4) then gives precisely the 
defining condition for a bosonic supersymmetric background of type I supergravity in ten dimensions.

To summarise, we have shown that any bosonic supersymmetric background of type I supergravity in ten dimensions can be embedded via (4.3) in a bosonic supersymmetric background of Poincaré supergravity in eleven dimensions, obeying (4.1) for some $\hat{\epsilon}=$ $\mathbf{I} \hat{\epsilon}$ and the second field equation in (4.2). Of course, the projection condition in eleven dimensions is because any background of type I supergravity in ten dimensions can preserve no more than sixteen real supercharges (in contrast with the maximum of thirty two in eleven dimensions).

\subsection{Embedding of maximally supersymmetric Freund-Rubin backgrounds}

Poincaré supergravity in eleven dimensions admits two well-known maximally supersymmetric Freund-Rubin solutions. In terms of the scalar curvature $\hat{R}$ of $\hat{g}$, they are of the form

- $A d S_{4}(8 \hat{R}) \times S^{7}(-7 \hat{R})$ with $\hat{F}=\sqrt{-6 \hat{R}} \operatorname{vol}_{A d S_{4}}($ if $\hat{R}<0)$.

- $A d S_{7}(-7 \hat{R}) \times S^{4}(8 \hat{R})$ with $\hat{F}=\sqrt{6 \hat{R}} \operatorname{vol}_{S^{4}}($ if $\hat{R}>0)$.

(The scalar curvature of each $A d S$ and $S$ factor is denoted in parenthesis.)

To make our description of the embedding as transparent as possible, let us adopt the following notation. Let $g_{n}$ denote the 'unit radius' metric on either $A d S_{n}$ or $S^{n}$ (i.e., the metric with constant scalar curvature $-n(n-1)$ for $A d S_{n}$ or $n(n-1)$ for $\left.S^{n}\right)$. Any metric of the form $\kappa^{2} g_{n}+\lambda^{2} g_{m}$ will be assumed to be Lorentzian (i.e., $A d S_{n} \times S^{m}$ or $S^{n} \times A d S_{m}$ ). Let $\operatorname{vol}_{n}$ denote the volume form with respect to $g_{n}$. Let $\psi_{n}$ denote a Killing spinor with respect to $g_{n}$, obeying $\nabla_{\mu} \psi_{n}= \pm \frac{1}{2} \Gamma_{\mu} \psi_{n}$ for $A d S_{n}$ or $\nabla_{\mu} \psi_{n}= \pm \frac{i}{2} \Gamma_{\mu} \psi_{n}$ for $S^{n}$. For $A d S_{n}$ (or $\left.S^{n}\right), \psi_{n}$ lifts to a constant spinor on the flat cone $C\left(A d S_{n}\right) \cong \mathbb{R}^{n-1,2}\left(\right.$ or $\left.C\left(S^{n}\right) \cong \mathbb{R}^{n+1}\right)$. We shall refer to $\psi_{n}$ as having unit Killing constant. Rescaling $g_{n}$ by a factor of $\kappa^{2}$ rescales the Killing constant by a factor of $\kappa^{-1}$.

In terms of this notation, the data for the maximally supersymmetric Freund-Rubin solutions of eleven-dimensional Poincaré supergravity is given by

$$
\hat{g}=\hat{\kappa}^{2}\left(g_{4}+4 g_{7}\right) \quad \text { and } \quad \hat{F}=3 \hat{\kappa}^{3} \operatorname{vol}_{4},
$$

while the supersymmetry parameter $\hat{\epsilon}$ involves a tensor product of $\psi_{4}$ (with Killing constant $\hat{\kappa}^{-1}$ ) and $\psi_{7}$ (with Killing constant $(2 \hat{\kappa})^{-1}$ ). The constant

$$
\hat{\kappa}:=\sqrt{\frac{3}{2|\hat{R}|}} .
$$

Observe that the factors of $\hat{\kappa}$ above are precisely the same as for the homothety noted at the end of section 4.1 , hence we can and will fix $\hat{\kappa}=1$ via the action of a homothety with $\alpha=\hat{\kappa}^{-1}$.

On the other hand, the data for the maximally supersymmetric Freund-Rubin backgrounds of ten-dimensional conformal supergravity is given by

$$
g=\kappa^{2}\left(g_{3}+4 g_{7}\right) \quad \text { and } \quad H=3 \kappa^{2} \operatorname{vol}_{3},
$$


while the supersymmetry parameter $\epsilon$ involves a tensor product of $\psi_{3}$ (with Killing constant $\kappa^{-1}$ ) and $\psi_{7}$ (with Killing constant $(2 \kappa)^{-1}$ ). The constant

$$
\kappa:=\sqrt{\frac{9}{2|R|}} .
$$

Since $G=0$ for this class of backgrounds, notice that the factors of $\kappa$ above are precisely the same as for a (constant) Weyl transformation. Therefore we shall fix $\kappa=1$ via a Weyl transformation with $\Omega=\kappa^{-1}$.

To embed (4.7) (with $\kappa=1$ ) in (4.5) (with $\hat{\kappa}=1$ ), it remains only to recognise the canonical 'equatorial' embedding defined by

$$
g_{4}=\mathrm{d} z^{2}+f(z)^{2} g_{3}
$$

where $f(z)$ is $\cosh (z)$ for $A d S_{3} \subset A d S_{4}$ or $\cos (z)$ for $S^{3} \subset S^{4}$, in terms of the 'colatitude' $z$. From (4.9), it follows that $\operatorname{vol}_{4}=f(z)^{3} \mathrm{~d} z \wedge \mathrm{vol}_{3}$ and hence that at $z=0$, we have

$$
\hat{g}=\mathrm{d} z^{2}+g \quad \text { and } \quad \hat{F}=\mathrm{d} z \wedge H .
$$

The embedding of the supersymmetry parameter $\epsilon$ in $\hat{\epsilon}$ is prescribed by the embedding of the unit Killing spinor $\psi_{3}$ in $\psi_{4}$ (the other Killing spinor $\psi_{7}$ clearly just goes along for the ride). Recall that $\psi_{3}$ and $\psi_{4}$ are completely specified by constant spinors on their respective (flat) cones. By definition, in terms of a radial coordinate $r$, the relevant cone metric $g_{C_{n+1}}$ is either $-\mathrm{d} r^{2}+r^{2} g_{n}$ for $A d S_{n}$ or $\mathrm{d} r^{2}+r^{2} g_{n}$ for $S^{n}$. For $A d S_{3} \subset A d S_{4}$, it follows that

$$
g_{C_{5}}=-\mathrm{d} r^{2}+r^{2} g_{4}=-\mathrm{d} r^{2}+r^{2} \mathrm{~d} z^{2}+(r \cosh (z))^{2} g_{3}=\mathrm{d} x^{2}-\mathrm{d} y^{2}+y^{2} g_{3}=\mathrm{d} x^{2}+g_{C_{4}},
$$

where $x=r \sinh (z)$ and $y=r \cosh (z)$. The embedding $\Delta_{+}^{(2,2)} \subset \Delta^{(3,2)}$ of Killing spinors here is therefore prescribed by restricting $\Delta^{(3,2)}$ to the $x=0$ hyperplane in $C_{5} \cong \mathbb{R}^{3,2}$. Similarly, for $S^{3} \subset S^{4}$, it follows that

$$
g_{C_{5}}=\mathrm{d} r^{2}+r^{2} g_{4}=\mathrm{d} r^{2}+r^{2} \mathrm{~d} z^{2}+(r \cos (z))^{2} g_{3}=\mathrm{d} x^{2}+\mathrm{d} y^{2}+y^{2} g_{3}=\mathrm{d} x^{2}+g_{C_{4}},
$$

where $x=r \sin (z)$ and $y=r \cos (z)$. Therefore the embedding $\Delta_{+}^{(4)} \subset \Delta^{(5)}$ of Killing spinors here is prescribed by restricting $\Delta^{(5)}$ to the $x=0$ hyperplane in $C_{5} \cong \mathbb{R}^{5}$.

\subsection{Branes, delocalisation and near-horizon limits}

Two well-known half-BPS solutions of supergravity in eleven dimensions are the M2-brane and the M5-brane.

The M2-brane solution has metric and four-form given by

$$
\hat{g}=f^{-2 / 3} g_{\mathbb{R}^{2,1}}+f^{1 / 3} g_{\mathbb{R}^{8}} \quad \text { and } \quad \hat{F}=\operatorname{vol}_{\mathbb{R}^{2,1}} \wedge \mathrm{d} f^{-1},
$$

where $f$ is a harmonic function on $\mathbb{R}^{8}$ so that the second field equation in (4.2) is satisfied (i.e., $\mathrm{d} \hat{\star} \hat{F}=0$ since $\hat{F} \wedge \hat{F}=0$ for (4.13)). The supersymmetry parameter is given by

$$
\hat{\epsilon}=f^{-1 / 6} \hat{\epsilon}_{0} \quad \text { with } \quad \operatorname{vol}_{\mathbb{R}^{2,1}} \hat{\epsilon}_{0}=\hat{\epsilon}_{0},
$$

where $\hat{\epsilon}_{0}$ is a constant Majorana spinor on $\mathbb{R}^{10,1}$. 
By identifying $z$ with a spatial coordinate on $\mathbb{R}^{2,1}$ and $f=\mathrm{e}^{-2 \Phi}$, one recognises that (4.13) is of the form (4.3). With respect to these identifications, the data $(g, H)$ in (4.3) gives precisely the F1-string background of type I supergravity in ten dimensions in (2.55). The conformally related data $\left(\mathrm{e}^{-2 \Phi / 3} g, \mathrm{e}^{-2 \Phi / 3} H\right)$ in ten dimensions gives precisely the half-BPS background of conformal supergravity with maximally supersymmetric $A d S_{3} \times S^{7}$ near-horizon limit. On the other hand, the near-horizon limit of (4.13) in eleven dimensions with $f=\mathrm{e}^{-2 \Phi}=1+\frac{\left|k_{2}\right|}{r^{6}}$ gives the maximally supersymmetric solution $A d S_{4}(8 \hat{R}) \times S^{7}(-7 \hat{R})$ with $\hat{F}=\sqrt{-6 \hat{R}} \operatorname{vol}_{A d S_{4}}$ (after identifying $\left|k_{2}\right|^{-1 / 3}=-\hat{R} / 6$ ).

The M5-brane solution has metric and four-form given by

$$
\hat{g}=f^{-1 / 3} g_{\mathbb{R}^{5,1}}+f^{2 / 3} g_{\mathbb{R}^{5}} \quad \text { and } \quad \hat{\star} \hat{F}=\operatorname{vol}_{\mathbb{R}^{5,1}} \wedge \mathrm{d} f^{-1},
$$

where $f$ is a harmonic function on $\mathbb{R}^{5}$ so that $\mathrm{d} \hat{F}=0$. The supersymmetry parameter is given by

$$
\hat{\epsilon}=f^{-1 / 12} \hat{\epsilon}_{0} \quad \text { with } \quad \operatorname{vol}_{\mathbb{R}^{5,1}} \hat{\epsilon}_{0}=\hat{\epsilon}_{0},
$$

where again $\hat{\epsilon}_{0}$ is a constant Majorana spinor on $\mathbb{R}^{10,1}$.

By identifying $z$ with a coordinate on $\mathbb{R}^{5}$ and $f=\mathrm{e}^{2 \Phi}$, one recognises that (4.15) is of the form (4.3). However, $\partial_{z}$ is a Killing vector only if $f$ is harmonic on the subspace $\mathbb{R}^{4} \subset \mathbb{R}^{5}$ orthogonal to the $z$-direction. Making this assumption is known as 'delocalisation' along the $z$-direction. With respect to these identifications, the data $(g, H)$ in (4.3) for the delocalised M5-brane gives precisely the NS5-brane background of type I supergravity in ten dimensions in (2.57). The near-horizon limit of the delocalised M5-brane (4.15) in eleven dimensions with $f=\mathrm{e}^{2 \Phi}=1+\frac{\left|k_{6}\right|}{r^{2}}$ defines a half-BPS background that is conformally equivalent to $\mathbb{R}^{5,1} \times H^{2} \times S^{3}$ (cf. $\mathbb{R}^{5,1} \times \mathbb{R}_{+} \times S^{3}$ in the near-horizon limit of the NS5-brane in ten dimensions). On the other hand, without delocalisation, the near-horizon limit of (4.15) with $f=\mathrm{e}^{2 \Phi}=1+\frac{\left|k_{6}^{\prime}\right|}{r^{3}}$ gives the maximally supersymmetric solution $A d S_{7}(-7 \hat{R}) \times S^{4}(8 \hat{R})$ with $\hat{F}=\sqrt{6 \hat{R}} \operatorname{vol}_{S^{4}}$ (after identifying the constant $k_{6}^{\prime}$ such that $\left|k_{6}^{\prime}\right|^{-2 / 3}=2 \hat{R} / 3$ ). Of course, without delocalisation, the ansatz (4.3) cannot be used to reduce to ten dimensions. Even so, notice that the data $\left(\mathrm{e}^{-2 \Phi / 3} g, \mathrm{e}^{-2 \Phi / 3} H\right)$ in ten dimensions obtained by comparing (4.3) with (4.15) without delocalisation gives precisely the half-BPS background of conformal supergravity with maximally supersymmetric $A d S_{7} \times S^{3}$ near-horizon limit.

\section{Acknowledgments}

The research of JMF is supported in part by the grant ST/J000329/1 "Particle Theory at the Tait Institute" from the U.K. Science and Technology Facilities Council, which also funded the visit of PdM to Edinburgh which started this collaboration. During the final stretch of writing, JMF was a guest of the Grupo de Investigación "Geometría diferencial y convexa" of the Universidad de Murcia, and he wishes to thank Ángel Ferrández Izquierdo for the invitation, the hospitality and for providing such a pleasant working atmosphere. 


\section{A Clifford algebra and spinors in ten dimensions}

In ten dimensions and in lorentzian signature, the 'mostly plus' and 'mostly minus' inner products result in isomorphic Clifford algebras. Indeed, as real associative algebras, the Clifford algebra $\mathrm{C} \ell(9,1) \cong \mathrm{C} \ell(1,9) \cong \operatorname{Mat}_{32}(\mathbb{R})$. We shall work with $\mathrm{C} \ell(9,1)$ in this paper, which is defined by the relation

$$
\Gamma_{\mu} \Gamma_{\nu}+\Gamma_{\nu} \Gamma_{\mu}=+2 \eta_{\mu \nu} \mathbb{1}
$$

where $\eta_{\mu \nu}=\operatorname{diag}(-1, \underbrace{+1, \ldots,+1}_{9})$ has mostly plus signature. In particular, $\left(\Gamma_{0}\right)^{2}=-\mathbb{1}$. It follows from the above isomorphism that $\mathrm{C} \ell(9,1)$ has a unique irreducible module up to equivalence: let's call it $\Delta^{(9,1)}$. As a representation of the $\operatorname{spin}$ group $\operatorname{Spin}(9,1) \subset \mathrm{C} \ell(9,1)$, $\Delta^{(9,1)}$ decomposes as a direct sum of two irreducible spinor representations $\Delta^{(9,1)}=$ $\Delta_{+}^{(9,1)} \oplus \Delta_{-}^{(9,1)}$, where the subspaces $\Delta_{ \pm}^{(9,1)} \subset \Delta^{(9,1)}$ correspond to the \pm 1 -eigenspaces of the idempotent 'chirality matrix' $\Gamma=-\Gamma_{0} \Gamma_{1} \ldots \Gamma_{9}$, which is not central in $\mathrm{C} \ell(9,1)$ but does commute with $\operatorname{Spin}(9,1)$. In physics parlance, elements of $\Delta^{(9,1)}$ are known as Majorana spinors and elements of $\Delta_{ \pm}^{(9,1)}$ are known as ( \pm chirality) Majorana-Weyl spinors.

There exists on $\Delta^{(9,1)}$ a unique (up to an overall scale) symplectic form $\boldsymbol{C}$, the so-called charge conjugation matrix, which obeys

$$
\boldsymbol{C}\left(\Gamma_{\mu} \psi, \chi\right)=-\boldsymbol{C}\left(\psi, \Gamma_{\mu} \chi\right)
$$

It follows that $\boldsymbol{C}$ is $\operatorname{Spin}(9,1)$-invariant and, in addition, that the chirality matrix $\Gamma$ is skew-symmetric. This means that $\Delta_{ \pm}^{(9,1)}$ are lagrangian subspaces, so $C$ pairs $\Delta_{+}^{(9,1)}$ nondegenerately with $\Delta_{-}^{(9,1)}$, thus providing an isomorphism $\Delta_{+}^{(9,1)} \cong\left(\Delta_{-}^{(9,1)}\right)^{*}$ of $\operatorname{Spin}(9,1)$ representations.

The Clifford algebra $\mathrm{C} \ell(9,1)$ inherits a filtration from the tensor algebra and the associated graded algebra is the exterior algebra of $\mathbb{R}^{9,1}$. A convenient (vector space) isomorphism is provided by the skewsymmetric products of the gamma matrices:

$$
\Gamma_{\mu_{1} \ldots \mu_{k}}=\Gamma_{\left[\mu_{1}\right.} \ldots \Gamma_{\left.\mu_{k}\right]}=\frac{1}{k !} \sum_{\sigma \in S_{k}}(-1)^{|\sigma|} \Gamma_{\mu_{\sigma(1)}} \ldots \Gamma_{\mu_{\sigma(k)}},
$$

for degree $k>0$ elements (i.e., unit-weight skewsymmetrisation of $k$ distinct degree-one basis elements) and the identity element $\mathbb{1}$ for $k=0$. These form a basis for $\mathrm{C} \ell(9,1)$.

Some useful identities which follow are

$$
\begin{aligned}
\Gamma^{\alpha} \Gamma_{\mu_{1} \ldots \mu_{k}} \Gamma_{\alpha} & =(-1)^{k}(10-2 k) \Gamma_{\mu_{1} \ldots \mu_{k}} \\
\Gamma^{\alpha \beta} \Gamma_{\mu_{1} \ldots \mu_{k}} \Gamma_{\alpha \beta} & =\left(10-(10-2 k)^{2}\right) \Gamma_{\mu_{1} \ldots \mu_{k}},
\end{aligned}
$$

and

$$
\Gamma_{\mu_{1} \ldots \mu_{k}} \Gamma=\sigma_{k-1} \frac{1}{(10-k) !} \varepsilon_{\mu_{1} \ldots \mu_{k} \nu_{k+1} \ldots \nu_{10}} \Gamma^{\nu_{k+1} \ldots \nu_{10}}
$$

where $\sigma_{k}=(-1)^{\left\lfloor\frac{k+1}{2}\right\rfloor}$ (i.e., $\sigma_{1}=\sigma_{2}=-1, \sigma_{k+2}=-\sigma_{k}$ ) and $\varepsilon_{01 \ldots 9}=+1$. 
Now let us write $\bar{\psi} \chi=\boldsymbol{C}(\psi, \chi)$, for any $\psi, \chi \in \Delta^{(9,1)}$. It follows that

$$
\bar{\psi} \Gamma_{\mu_{1} \ldots \mu_{k}} \chi=-\sigma_{k} \bar{\chi} \Gamma_{\mu_{1} \ldots \mu_{k}} \psi
$$

and

$$
\bar{\psi}_{ \pm} \Gamma_{\mu_{1} \ldots \mu_{2 k}} \chi_{ \pm}=0
$$

for any $\psi_{ \pm}, \chi_{ \pm} \in \Delta_{ \pm}^{(9,1)}$. Furthermore, we have the Fierz identities

$$
\begin{aligned}
& \psi_{ \pm} \bar{\chi}_{ \pm}=\frac{1}{32}\left(2\left(\bar{\chi}_{ \pm} \Gamma^{\mu} \psi_{ \pm}\right) \Gamma_{\mu}-\frac{1}{3}\left(\bar{\chi}_{ \pm} \Gamma^{\mu \nu \rho} \psi_{ \pm}\right) \Gamma_{\mu \nu \rho}+\frac{1}{5 !}\left(\bar{\chi}_{ \pm} \Gamma^{\mu \nu \rho \sigma \tau} \psi_{ \pm}\right) \Gamma_{\mu \nu \rho \sigma \tau}\right) \Pi_{\mp} \\
& \psi_{ \pm} \bar{\chi}_{\mp}=\frac{1}{16}\left(\left(\bar{\chi}_{\mp} \psi_{ \pm}\right) \mathbb{1}-\frac{1}{2}\left(\bar{\chi}_{\mp} \Gamma^{\mu \nu} \psi_{ \pm}\right) \Gamma_{\mu \nu}+\frac{1}{4 !}\left(\bar{\chi}_{\mp} \Gamma^{\mu \nu \rho \sigma} \psi_{ \pm}\right) \Gamma_{\mu \nu \rho \sigma}\right) \Pi_{ \pm}
\end{aligned}
$$

where $\boldsymbol{\Pi}_{ \pm}=\frac{1}{2}(\mathbb{1} \pm \Gamma)$. The bilinear $\bar{\chi}_{ \pm} \Gamma_{\mu \nu \rho \sigma \tau} \psi_{ \pm}$defines a five-form that is self-dual if the spinors have positive chirality and anti-self-dual if the spinors have negative chirality.

It follows from (A.6) and (A.7) that, if $\epsilon \in \Delta_{+}^{(9,1)}$, all bilinears built from $\epsilon$ vanish identically except for $\xi_{\mu}=\bar{\epsilon} \Gamma_{\mu} \epsilon$ and $\zeta_{\mu \nu \rho \sigma \tau}=\bar{\epsilon} \Gamma_{\mu \nu \rho \sigma \tau} \epsilon$, which are nonzero for nonzero $\epsilon$. The Fierz identity (A.8) for $\epsilon$ reads

$$
\epsilon \bar{\epsilon}=\frac{1}{32}(2 \xi+\zeta) \Pi_{-},
$$

and a useful subsidiary identity is

$$
-\Gamma^{\mu \nu} \epsilon \bar{\epsilon} \Gamma_{\nu}=\left(\left(\epsilon \bar{\epsilon}-\frac{1}{2} \xi\right) \Gamma^{\mu}+\xi^{\mu} \mathbb{1}\right) \Pi_{+} .
$$

An unrelated source of \pm signs comes from choosing a Witt (or 'lightcone') basis for $\mathbb{R}^{9,1}$. We choose a somewhat asymmetrical definition:

$$
\Gamma_{+}:=\frac{1}{2}\left(\Gamma_{9}+\Gamma_{0}\right) \quad \text { and } \quad \Gamma_{-}:=\Gamma_{9}-\Gamma_{0} .
$$

It follows that $\Gamma_{ \pm}^{2}=0$ and that

$$
\Gamma_{+} \Gamma_{-}+\Gamma_{-} \Gamma_{+}=2 \mathbb{1}
$$

This last identity means that we may decompose $\Delta_{+}^{(9,1)}$ into the direct sum of the two subspaces $\operatorname{ker} \Gamma_{ \pm}: \Delta_{+}^{(9,1)} \rightarrow \Delta_{-}^{(9,1)}$, with $\frac{1}{2} \Gamma_{ \pm} \Gamma_{\mp}$ the corresponding projectors.

Open Access. This article is distributed under the terms of the Creative Commons Attribution License (CC-BY 4.0), which permits any use, distribution and reproduction in any medium, provided the original author(s) and source are credited. 


\section{References}

[1] G.F. Chapline and N.S. Manton, Unification of Yang-Mills Theory and Supergravity in Ten-Dimensions, Phys. Lett. B 120 (1983) 105 [InSPIRE].

[2] I.C.G. Campbell and P.C. West, $N=2 D=10$ nonchiral supergravity and its spontaneous compactification, Nucl. Phys. B 243 (1984) 112 [INSPIRE].

[3] F. Giani and M. Pernici, $N=2$ supergravity in ten-dimensions, Phys. Rev. D 30 (1984) 325 [INSPIRE].

[4] M. Huq and M.A. Namazie, Kaluza-Klein supergravity in ten-dimensions, Class. Quant. Grav. 2 (1985) 293 [Erratum ibid. 2 (1985) 597] [INSPIRE].

[5] L. Romans, Massive $N=2 a$ supergravity in ten dimensions, Phys. Lett. B 169 (1986) 374.

[6] J.H. Schwarz and P.C. West, Symmetries and transformations of chiral $N=2 D=10$ supergravity, Phys. Lett. B 126 (1983) 301 [INSPIRE].

[7] J.H. Schwarz, Covariant field equations of chiral $N=2 D=10$ supergravity, Nucl. Phys. B 226 (1983) 269 [InSPIRE].

[8] P.S. Howe and P.C. West, The complete $N=2, D=10$ supergravity, Nucl. Phys. B 238 (1984) 181 [InSPIRE].

[9] E. Bergshoeff, M. de Roo and B. de Wit, Conformal supergravity in ten-dimensions, Nucl. Phys. B 217 (1983) 489 [inSPIRE].

[10] W. Nahm, Supersymmetries and their representations, Nucl. Phys. B 135 (1978) 149 [INSPIRE].

[11] A. Altomani and A. Santi, Classification of maximal transitive prolongations of super-Poincaré algebras, Adv. Math. 265 (2014) 60 [arXiv:1212.1826].

[12] J.W. van Holten and A. Van Proeyen, $N=1$ Supersymmetry Algebras in $d=2,3,4$ mod-8, J. Phys. A 15 (1982) 3763 [inSPIRE].

[13] M. Blau, Killing spinors and SYM on curved spaces, JHEP 11 (2000) 023 [hep-th/0005098] [INSPIRE].

[14] V. Pestun, Localization of gauge theory on a four-sphere and supersymmetric Wilson loops, Commun. Math. Phys. 313 (2012) 71 [arXiv:0712.2824] [InSPIRE].

[15] G. Festuccia and N. Seiberg, Rigid Supersymmetric Theories in Curved Superspace, JHEP 06 (2011) 114 [arXiv:1105.0689] [INSPIRE].

[16] B. Jia and E. Sharpe, Rigidly Supersymmetric Gauge Theories on Curved Superspace, JHEP 04 (2012) 139 [arXiv:1109.5421] [INSPIRE].

[17] H. Samtleben and D. Tsimpis, Rigid supersymmetric theories in 4 d Riemannian space, JHEP 05 (2012) 132 [arXiv:1203.3420] [INSPIRE].

[18] C. Klare, A. Tomasiello and A. Zaffaroni, Supersymmetry on Curved Spaces and Holography, JHEP 08 (2012) 061 [arXiv: 1205.1062] [INSPIRE].

[19] T.T. Dumitrescu, G. Festuccia and N. Seiberg, Exploring Curved Superspace, JHEP 08 (2012) 141 [arXiv:1205.1115] [INSPIRE]. 
[20] D. Cassani, C. Klare, D. Martelli, A. Tomasiello and A. Zaffaroni, Supersymmetry in Lorentzian Curved Spaces and Holography, Commun. Math. Phys. 327 (2014) 577 [arXiv: 1207.2181] [INSPIRE].

[21] J.T. Liu, L.A. Pando Zayas and D. Reichmann, Rigid Supersymmetric Backgrounds of Minimal Off-Shell Supergravity, JHEP 10 (2012) 034 [arXiv:1207.2785] [INSPIRE].

[22] K. Ito, H. Nakajima and S. Sasaki, Torsion and Supersymmetry in Omega-background, JHEP 11 (2012) 132 [arXiv:1209.2561] [InSPIRE].

[23] P. de Medeiros, Rigid supersymmetry, conformal coupling and twistor spinors, JHEP 09 (2014) 032 [arXiv: 1209.4043] [INSPIRE].

[24] M. Fujitsuka, M. Honda and Y. Yoshida, Maximal super Yang-Mills theories on curved background with off-shell supercharges, JHEP 01 (2013) 162 [arXiv:1209.4320] [INSPIRE].

[25] T.T. Dumitrescu and G. Festuccia, Exploring Curved Superspace (II), JHEP 01 (2013) 072 [arXiv: 1209.5408] [INSPIRE].

[26] A. Kehagias and J.G. Russo, Global Supersymmetry on Curved Spaces in Various Dimensions, Nucl. Phys. B 873 (2013) 116 [arXiv:1211.1367] [INSPIRE].

[27] C. Closset, T.T. Dumitrescu, G. Festuccia and Z. Komargodski, Supersymmetric Field Theories on Three-Manifolds, JHEP 05 (2013) 017 [arXiv: 1212.3388] [INSPIRE].

[28] D. Martelli, A. Passias and J. Sparks, The supersymmetric NUTs and bolts of holography, Nucl. Phys. B 876 (2013) 810 [arXiv:1212.4618] [INSPIRE].

[29] H. Samtleben, E. Sezgin and D. Tsimpis, Rigid 6D supersymmetry and localization, JHEP 03 (2013) 137 [arXiv:1212.4706] [InSPIRE].

[30] S.M. Kuzenko, Symmetries of curved superspace, JHEP 03 (2013) 024 [arXiv:1212.6179] [INSPIRE].

[31] K. Hristov, A. Tomasiello and A. Zaffaroni, Supersymmetry on Three-dimensional Lorentzian Curved Spaces and Black Hole Holography, JHEP 05 (2013) 057 [arXiv:1302.5228] [INSPIRE].

[32] P. de Medeiros and S. Hollands, Conformal symmetry superalgebras, Class. Quant. Grav. 30 (2013) 175016 [arXiv:1302.7269] [INSPIRE].

[33] P. de Medeiros and S. Hollands, Superconformal quantum field theory in curved spacetime, Class. Quant. Grav. 30 (2013) 175015 [arXiv:1305.0499] [INSPIRE].

[34] D. Martelli and A. Passias, The gravity dual of supersymmetric gauge theories on a two-parameter deformed three-sphere, Nucl. Phys. B 877 (2013) 51 [arXiv:1306.3893] [INSPIRE].

[35] D. Cassani and D. Martelli, Supersymmetry on curved spaces and superconformal anomalies, JHEP 10 (2013) 025 [arXiv: 1307.6567] [INSPIRE].

[36] L.F. Alday, D. Martelli, P. Richmond and J. Sparks, Localization on Three-Manifolds, JHEP 10 (2013) 095 [arXiv: 1307.6848] [InSPIRE].

[37] K. Ito, H. Nakajima and S. Sasaki, Twisted $\mathcal{N}=4$ Super Yang-Mills Theory in $\Omega$-background, JHEP 10 (2013) 080 [arXiv:1307.7565] [INSPIRE].

[38] S.M. Kuzenko, Super-Weyl anomalies in $N=2$ supergravity and (non)local effective actions, JHEP 10 (2013) 151 [arXiv:1307.7586] [INSPIRE]. 
[39] C. Klare and A. Zaffaroni, Extended Supersymmetry on Curved Spaces, JHEP 10 (2013) 218 [arXiv:1308.1102] [INSPIRE].

[40] Y. Pan, Rigid Supersymmetry on 5-dimensional Riemannian Manifolds and Contact Geometry, JHEP 05 (2014) 041 [arXiv:1308.1567] [INSPIRE].

[41] C. Closset, T.T. Dumitrescu, G. Festuccia and Z. Komargodski, The Geometry of Supersymmetric Partition Functions, JHEP 01 (2014) 124 [arXiv:1309.5876] [INSPIRE].

[42] T. Nosaka and S. Terashima, Supersymmetric Gauge Theories on a Squashed Four-Sphere, JHEP 12 (2013) 001 [arXiv:1310.5939] [INSPIRE].

[43] C. Closset and I. Shamir, The $\mathcal{N}=1$ Chiral Multiplet on $T^{2} \times S^{2}$ and Supersymmetric Localization, JHEP 03 (2014) 040 [arXiv:1311.2430] [INSPIRE].

[44] J. Figueroa-O'Farrill and M. Gharamti, Supersymmetry of hyperbolic monopoles, JHEP 04 (2014) 074 [arXiv:1311.3588] [INSPIRE].

[45] N.S. Deger, A. Kaya, H. Samtleben and E. Sezgin, Supersymmetric Warped AdS in Extended Topologically Massive Supergravity, Nucl. Phys. B 884 (2014) 106 [arXiv: 1311.4583] [INSPIRE].

[46] S.M. Kuzenko, U. Lindström, M. Roček, I. Sachs and G. Tartaglino-Mazzucchelli, Three-dimensional $\mathcal{N}=2$ supergravity theories: from superspace to components, Phys. Rev. D 89 (2014) 085028 [arXiv:1312.4267] [INSPIRE].

[47] D. Cassani and D. Martelli, The gravity dual of supersymmetric gauge theories on a squashed $S^{1} \times S^{3}$, JHEP 08 (2014) 044 [arXiv: 1402.2278] [INSPIRE].

[48] L. Di Pietro, M. Dine and Z. Komargodski, (Non-)Decoupled Supersymmetric Field Theories, JHEP 04 (2014) 073 [arXiv: 1402.3385] [INSPIRE].

[49] S.M. Kuzenko and G. Tartaglino-Mazzucchelli, $N=4$ supersymmetric Yang-Mills theories in $A d S_{3}, J H E P 05$ (2014) 018 [arXiv:1402.3961] [INSPIRE].

[50] T. Anous, D.Z. Freedman and A. Maloney, de Sitter Supersymmetry Revisited, JHEP 07 (2014) 119 [arXiv: 1403.5038] [INSPIRE].

[51] Y. Imamura and H. Matsuno, Supersymmetric backgrounds from $5 d N=1$ supergravity, JHEP 07 (2014) 055 [arXiv: 1404.0210] [InSPIRE].

[52] D. Farquet, J. Lorenzen, D. Martelli and J. Sparks, Gravity duals of supersymmetric gauge theories on three-manifolds, arXiv: 1404.0268 [INSPIRE].

[53] L.F. Alday, M. Fluder, P. Richmond and J. Sparks, Gravity dual of supersymmetric gauge theories on a squashed five-sphere, Phys. Rev. Lett. 113 (2014) 141601 [arXiv:1404.1925] [INSPIRE].

[54] B. Assel, D. Cassani and D. Martelli, Localization on Hopf surfaces, JHEP 08 (2014) 123 [arXiv: 1405.5144] [INSPIRE].

[55] L.F. Alday, M. Fluder, C.M. Gregory, P. Richmond and J. Sparks, Supersymmetric gauge theories on squashed five-spheres and their gravity duals, JHEP 09 (2014) 067 [arXiv: 1405.7194] [INSPIRE].

[56] S.M. Kuzenko, J. Novak and G. Tartaglino-Mazzucchelli, Symmetries of curved superspace in five dimensions, JHEP 10 (2014) 175 [arXiv: 1406.0727] [INSPIRE].

[57] D. Farquet and J. Sparks, Wilson loops on three-manifolds and their M2-brane duals, JHEP 12 (2014) 173 [arXiv:1406.2493] [INSPIRE]. 
[58] P. de Medeiros, Submaximal conformal symmetry superalgebras for Lorentzian manifolds of low dimension, JHEP 02 (2016) 008 [arXiv: 1406.5166] [INSPIRE].

[59] Y. Pan, 5d Higgs Branch Localization, Seiberg-Witten Equations and Contact Geometry, JHEP 01 (2015) 145 [arXiv:1406.5236] [INSPIRE].

[60] D. Butter, New approach to curved projective superspace, Phys. Rev. D 92 (2015) 085004 [arXiv:1406.6235] [INSPIRE].

[61] S.M. Kuzenko and D. Sorokin, Superconformal structures on the three-sphere, JHEP 10 (2014) 80 [arXiv: 1406.7090] [INSPIRE].

[62] C. Closset, T.T. Dumitrescu, G. Festuccia and Z. Komargodski, From Rigid Supersymmetry to Twisted Holomorphic Theories, Phys. Rev. D 90 (2014) 085006 [arXiv:1407.2598] [INSPIRE].

[63] L. Di Pietro and Z. Komargodski, Cardy formulae for SUSY theories in $d=4$ and $d=6$, JHEP 12 (2014) 031 [arXiv:1407.6061] [INSPIRE].

[64] T. Nishioka and I. Yaakov, Generalized indices for $\mathcal{N}=1$ theories in four-dimensions, JHEP 12 (2014) 150 [arXiv: 1407.8520] [INSPIRE].

[65] A. Lischewski, Conformal superalgebras via tractor calculus, Class. Quantum Grav. 32 (2015) 015020 [arXiv:1408.2238].

[66] J. Gomis and N. Ishtiaque, Kähler potential and ambiguities in $4 d \mathcal{N}=2$ SCFTs, JHEP 04 (2015) 169 [arXiv: 1409.5325] [INSPIRE].

[67] Y. Yoshida and K. Sugiyama, Localization of $3 d \mathcal{N}=2$ Supersymmetric Theories on $S^{1} \times D^{2}$, arXiv: 1409.6713 [INSPIRE].

[68] B. Assel, D. Cassani and D. Martelli, Supersymmetric counterterms from new minimal supergravity, JHEP 11 (2014) 135 [arXiv:1410.6487] [INSPIRE].

[69] D. Butter, S.M. Kuzenko, J. Novak and G. Tartaglino-Mazzucchelli, Conformal supergravity in five dimensions: new approach and applications, JHEP 02 (2015) 111 [arXiv: 1410.8682] [INSPIRE].

[70] A. Bawane, G. Bonelli, M. Ronzani and A. Tanzini, $\mathcal{N}=2$ supersymmetric gauge theories on $S^{2} \times S^{2}$ and Liouville Gravity, JHEP 07 (2015) 054 [arXiv:1411.2762] [INSPIRE].

[71] M. Sinamuli, On $N=2$ supersymmetric gauge theories on $S^{2} \times S^{2}$, arXiv: 1411.4918 [INSPIRE].

[72] D. Rodriguez-Gomez and J. Schmude, Partition functions for equivariantly twisted $\mathcal{N}=2$ gauge theories on toric Kähler manifolds, JHEP 05 (2015) 111 [arXiv:1412.4407] [INSPIRE].

[73] G. Knodel, J.T. Liu and L.A. Pando Zayas, On $N=1$ partition functions without R-symmetry, JHEP 03 (2015) 132 [arXiv:1412.4804] [INSPIRE].

[74] A. Cabo-Bizet, E. Gava, V.I. Giraldo-Rivera, M.N. Muteeb and K.S. Narain, Partition Function of $N=2$ Gauge Theories on a Squashed $S^{4}$ with $\mathrm{SU}(2) \times \mathrm{U}(1)$ Isometry, Nucl. Phys. B 899 (2015) 149 [arXiv:1412.6826] [INSPIRE].

[75] J. Lorenzen and D. Martelli, Comments on the Casimir energy in supersymmetric field theories, JHEP 07 (2015) 001 [arXiv: 1412.7463] [INSPIRE].

[76] J.A. Minahan and M. Zabzine, Gauge theories with 16 supersymmetries on spheres, JHEP 03 (2015) 155 [arXiv: 1502.07154] [INSPIRE]. 
[77] B. Assel, D. Cassani, L. Di Pietro, Z. Komargodski, J. Lorenzen and D. Martelli, The Casimir Energy in Curved Space and its Supersymmetric Counterpart, JHEP 07 (2015) 043 [arXiv: 1503.05537] [INSPIRE].

[78] L.F. Alday, P.B. Genolini, M. Fluder, P. Richmond and J. Sparks, Supersymmetric gauge theories on five-manifolds, JHEP 08 (2015) 007 [arXiv:1503.09090] [INSPIRE].

[79] Y. Pan and J. Schmude, On rigid supersymmetry and notions of holomorphy in five dimensions, JHEP 11 (2015) 041 [arXiv: 1504.00321] [INSPIRE].

[80] F. Benini and A. Zaffaroni, A topologically twisted index for three-dimensional supersymmetric theories, JHEP 07 (2015) 127 [arXiv: 1504.03698] [INSPIRE].

[81] A. Pini, D. Rodriguez-Gomez and J. Schmude, Rigid Supersymmetry from Conformal Supergravity in Five Dimensions, JHEP 09 (2015) 118 [arXiv: 1504.04340] [INSPIRE].

[82] M. Honda and Y. Yoshida, Supersymmetric index on $T^{2} \times S^{2}$ and elliptic genus, arXiv: 1504.04355 [INSPIRE].

[83] S.M. Kuzenko, Supersymmetric Spacetimes from Curved Superspace, PoS (CORFU2014) 140 [arXiv:1504.08114] [INSPIRE].

[84] D. Butter, G. Inverso and I. Lodato, Rigid $4 D \mathcal{N}=2$ supersymmetric backgrounds and actions, JHEP 09 (2015) 088 [arXiv: 1505.03500] [INSPIRE].

[85] L.F. Alday, M. Fluder, C.M. Gregory, P. Richmond and J. Sparks, Supersymmetric solutions to Euclidean Romans supergravity, JHEP 02 (2016) 100 [arXiv: 1505.04641] [INSPIRE].

[86] T. Kawano and N. Matsumiya, 5D SYM on 3D Deformed Spheres, Nucl. Phys. B 898 (2015) 456 [arXiv:1505.06565] [InSPIRE].

[87] M. Fluder and J. Sparks, D2-brane Chern-Simons theories: F-maximization = a-maximization, JHEP 01 (2016) 048 [arXiv:1507.05817] [INSPIRE].

[88] D. Cassani, J. Lorenzen and D. Martelli, Comments on supersymmetric solutions of minimal gauged supergravity in five dimensions, arXiv:1510.01380 [INSPIRE].

[89] J. Qiu, L. Tizzano, J. Winding and M. Zabzine, Modular properties of full 5D SYM partition function, arXiv:1511.06304 [INSPIRE].

[90] D. Martelli and J. Sparks, The character of the supersymmetric Casimir energy, arXiv: 1512.02521 [INSPIRE].

[91] O. Aharony, M. Berkooz, A. Karasik and T. Vaknin, Supersymmetric Field Theories on $A d S_{p} \times S^{q}, \operatorname{arXiv}: 1512.04698$ [INSPIRE].

[92] J.A. Minahan, Localizing gauge theories on $S^{d}$, arXiv:1512.06924 [INSPIRE].

[93] A. Lischewski, Supersymmetric gauge theory on a class of cocalibrated $G_{2}$-structures, Class. Quant. Grav. 32 (2015) 115003.

[94] N. Berkovits, A ten-dimensional super Yang-Mills action with off-shell supersymmetry, Phys. Lett. B 318 (1993) 104 [hep-th/9308128] [INSPIRE].

[95] J.M. Evans, Supersymmetry algebras and Lorentz invariance for $d=10$ super Yang-Mills, Phys. Lett. B 334 (1994) 105 [hep-th/9404190] [INSPIRE].

[96] L. Baulieu, N.J. Berkovits, G. Bossard and A. Martin, Ten-dimensional super-Yang-Mills with nine off-shell supersymmetries, Phys. Lett. B 658 (2008) 249 [arXiv:0705.2002] [INSPIRE]. 
[97] J. Figueroa-O'Farrill and N. Hustler, The homogeneity theorem for supergravity backgrounds, JHEP 10 (2012) 014 [arXiv:1208.0553] [INSPIRE].

[98] U. Gran, G. Papadopoulos, D. Roest and P. Sloane, Geometry of all supersymmetric type-I backgrounds, JHEP 08 (2007) 074 [hep-th/0703143] [INSPIRE].

[99] B.S. Acharya, J.M. Figueroa-O'Farrill and B.J. Spence, Planes, branes and automorphisms. 1. Static branes, JHEP 07 (1998) 004 [hep-th/9805073] [INSPIRE].

[100] J.M. Figueroa-O'Farrill and G. Papadopoulos, Maximally supersymmetric solutions of ten-dimensional and eleven-dimensional supergravities, JHEP 03 (2003) 048 [hep-th/0211089] [INSPIRE].

[101] P. Griffiths and J. Harris, Principles of algebraic geometry, Wiley-Interscience, New York U.S.A. (1978).

[102] A.L. Besse, Einstein manifolds, in Ergebnisse der Mathematik und ihrer Grenzgebiete, vol. 10, Springer-Verlag, Berlin Germany (1987).

[103] M. Blau, J.M. Figueroa-O'Farrill and G. Papadopoulos, Penrose limits, supergravity and brane dynamics, Class. Quant. Grav. 19 (2002) 4753 [hep-th/0202111] [InSPIRE].

[104] J.M. Figueroa-O'Farrill, On the supersymmetries of Anti-de Sitter vacua, Class. Quant. Grav. 16 (1999) 2043 [hep-th/9902066] [INSPIRE].

[105] V. Kac, Classification of simple Lie superalgebras, Funct. Anal. Appl. 9 (1975) 91.

[106] M. Parker, Classification of real simple Lie superalgebras of classical type, J. Math. Phys. 21 (1980) 689.

[107] M. Hatsuda, K. Kamimura and M. Sakaguchi, Super-pp-wave algebra from super-AdS $\times S$ algebras in eleven-dimensions, Nucl. Phys. B 637 (2002) 168 [hep-th/0204002] [InSPIRE].

[108] M. Blau, J.M. Figueroa-O'Farrill, C. Hull and G. Papadopoulos, A new maximally supersymmetric background of IIB superstring theory, JHEP 01 (2002) 047 [hep-th/0110242] [INSPIRE].

[109] M. Blau, J.M. Figueroa-O'Farrill, C. Hull and G. Papadopoulos, Penrose limits and maximal supersymmetry, Class. Quant. Grav. 19 (2002) L87 [hep-th/0201081] [INSPIRE].

[110] J. Figueroa-O'Farrill, E. Hackett-Jones, G. Moutsopoulos and J. Simón, On the maximal superalgebras of supersymmetric backgrounds, Class. Quant. Grav. 26 (2009) 035016 [arXiv:0809.5034] [INSPIRE].

[111] P.K. Townsend, $M$ theory from its superalgebra, in Strings, branes and dualities. Proceedings, NATO Advanced Study Institute, Cargese, France, 26 May - 14 June 1997 [hep-th/9712004] [INSPIRE].

[112] K. Kamimura and M. Sakaguchi, osp(1|32) and extensions of super-AdS $S_{5} \times S^{5}$ algebra, Nucl. Phys. B 662 (2003) 491 [hep-th/0301083] [INSPIRE].

[113] A. Dabholkar, G.W. Gibbons, J.A. Harvey and F. Ruiz Ruiz, Superstrings and solitons, Nucl. Phys. B 340 (1990) 33 [INSPIRE].

[114] M.J. Duff and J.X. Lu, Elementary five-brane solutions of $D=10$ supergravity, Nucl. Phys. B 354 (1991) 141 [inSPIRE]. 